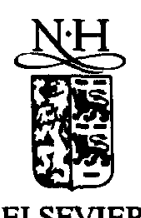

ELSEVIER

Applied Surface Science 92 (1996) 237-256

\title{
Atomistic dynamics of interfacial processes: films, junctions and nanostructures
}

\author{
Uzi Landman *, W.D. Luedtke \\ School of Physics, Georgia Institute of Technology, Atlanta, GA 30332, USA
}

Received 12 December 1994; accepted for publication 4 July 1995

\begin{abstract}
Studies of atomistic mechanisms, energetics and dynamics of surface and interfacial processes using computer-based molecular dynamics simulations employing realistic interaction potentials, open new avenues in investigations of basic and technological problems. Recent results of such studies are discussed for. surface processing via laser irradiation, superheating, melting, and annealing; stability, collapse and cooperative transport mechanisms of metallic nanostructures on surfaces; formation of interfacial solid and liquid junctions and their mechanical and electrical properties.
\end{abstract}

\section{Introduction}

Understanding the atomistic mechanisms, energetics and dynamics underlying interfacial interactions and physical processes occurring at interfaces is fundamentally important in basic research and is a key to the science base of many venerable technological problems (such as catalysis, liquid and vapor phase epitaxial growth, thin films and coatings, electrochemical processes, adhesion, contact formation, friction and lubrication, wear, fracture, nanoindentation, surface phase transformations, and surface processing, modification, manipulation and patterning). The quest to understand and observe natural phenomena on refined microscopic scales has led to the development of conceptual and technological devices allowing the interrogation of materials with increasing resolution. On the experimental front, the recent

\footnotetext{
${ }^{*}$ Corresponding author. Fax: + 1-404-853-9958.
}

emergence and proliferation of the surface force apparatus (SFA) [1,2], of scanning tunneling microscopy (STM) $[3,4]$, and of the related atomic force microscopy (AFM) [4-6], supplementing and complementing the wide arsenal of established surface science techniques, broaden our perspectives and abilities to probe the morphology, electronic structure, and nature of interfacial interatomic interactions in materials, as well as enhance our ability to manipulate materials surfaces on the atomic scale [7].

On the theoretical front, recent advances in the formulation and evaluation of the energetics and interatomic interactions in materials, coupled with the development and implementation of computational methods and simulation techniques (classical and quantum mechanical) open new avenues for investigations of the microscopic origins of complex materials phenomena [8,9]. In particular, large-scale molecular dynamics (MD) computer simulations, which are in a sense computer experiments where the evolution of a system of interacting particles is 
simulated with high spatial and temporal resolution by means of direct integration of the particles' equations of motion, have greatly enhanced our understanding of a broad range of materials phenomena.

In this paper we review selected case studies which demonstrate current advances in computer simulations of interfacial phenomena pertaining to: (a) surface processing via laser irradiation, superheating, melting and annealing [10]; (b) stability and collapse mechanisms of metallic nanostructures on surfaces [11,12], and (c) formation and properties of interfacial junctions [13-19]. For a review of atomistic simulations of liquid and vapor phase epitaxial growth see Ref. [12].

\section{Case studies}

\subsection{Superheating, melting and annealing of metal surfaces}

Methods of processing of materials surfaces via laser irradiation are of fundamental as well as technological and economical significance, since various physical and chemical surface properties (such as optical and electrical characteristics, corrosion resistance, composition, morphology, and structure) can be influenced and/or modified using such methods, in a controlled and spatially selective manner [20,21]. Furthermore, with the use of short laser pulses, detailed time-resolved experimental investigations of electron-phonon energy transfer mechanisms and relaxation times [23], and of the thermal and structural evolution of metal surfaces (disordering, melting [23a], and most recently superheating of the $\mathrm{Pb}$ (111) surface [23b]), have been performed. In these experiments it was found, using time-resolved reflection high-energy diffraction (RHEED) that during and following a laser pulse irradiation the surface superheated up to $120 \mathrm{~K}$ above the bulk melting temperature (temperatures were determined from Debye-Waller analysis of the RHEED intensities) while maintaining crystalline order.

Commonly laser processing of solid surfaces is discussed in the context of laser heating, melting, and subsequent solidification (or recrystallization) of the surface region of the sample [20,21]. Although the phenomenon of surface melting was proposed over a century ago [24] it is only during the past decade that controlled experimental observations and theoretical studies have been made [25-27] of surface disordering, roughening, melting and premelting (that is formation of a quasi-liquid surface region for $T<T_{\mathrm{m}}^{\mathrm{b}}$, where $T_{\mathrm{m}}^{\mathrm{b}}$ is the bulk melting temperature of the material). Both experimental $[23,25]$ and theoretical $[26,27]$ studies have shown that premelting can occur for the (110) surfaces of certain fcc metals (e.g., $\mathrm{Pb}(110) \quad[23 \mathrm{a}, 25,27 \mathrm{~d}], \mathrm{Al}(110) \quad[27 \mathrm{a}]$ and $\mathrm{Cu}(110)[26,27 \mathrm{~b}]$, while $\mathrm{Ni}(110)$ [27c], as well as the close-packed (111) surfaces of fcc metals do not premelt $[25,27 \mathrm{~b}])$, with the premelting propensity determined by the sign and magnitude of the interfacial free energy difference, $\Delta \gamma$, between the ordered solid surface and the surface wetted by its melt ( $\Delta \gamma>0$ corresponds to premelting tendency, thus precluding superheating for surfaces with $\Delta \gamma>0$ ). Furthermore, molecular dynamics (MD) simulations $[26,27]$ show that the nucleation mechanisms of the premelting process involves generation of vacancyadlayer atom pairs, with the energy required for such processes significantly higher at close-packed fcc metal surfaces than at the (110) surface.

To simulate the dynamics of copper surfaces irradiated by a laser pulse we developed [10] a MD method where the laser pulse deposits energy into the electronic subsystem, modeled as a uniform fluid, which couples locally to the ionic degrees of freedom whose dynamics is governed by many-body embedded-atom (EAM) potentials [28]. In a continuum model of the system (electronic and ionic) the time evolution of the electronic and lattice temperatures $\left(T_{\mathrm{e}}(z, t)\right.$ and $\left.T_{1}(z, t)\right)$ are described by two coupled nonlinear differential equations [29] (in conjunction with appropriate boundary conditions)

$$
\begin{aligned}
& C_{\mathrm{e}}\left(T_{\mathrm{e}}\right) \frac{\partial T_{\mathrm{e}}}{\partial t}=\kappa_{\mathrm{e}} \frac{\partial^{2} T_{\mathrm{e}}}{\partial z^{2}}-G\left(T_{\mathrm{e}}-T_{1}\right)+P(z, t), \\
& C_{1}\left(T_{1}\right) \frac{\partial T_{1}}{\partial t}=\kappa_{1} \frac{\partial^{2} T_{1}}{\partial z^{2}}+G\left(T_{\mathrm{e}}-T_{1}\right),
\end{aligned}
$$

where the characteristics of the laser pulse are given by

$P(z, t)=I_{0}(1-R) \alpha \mathrm{e}^{-\alpha z} f\left(t-t_{0}\right)$,

with a peak intensity $I_{0}$, an absorption coefficient $\alpha$, a reflectivity $R$, and a Gaussian temporal shape 
profile $f\left(t-t_{0}\right)=\exp \left[-\left(t-t_{0}\right)^{2} / 2 \sigma^{2}\right]$, with the FWHM given by $2 \sigma$. In the above equations $C_{\mathrm{e}}, \kappa_{\mathrm{e}}$ and $C_{1}, \kappa_{1}$ are the heat capacities and thermal conductivities for the electronic and lattice subsystems, respectively, $G$ is the electron-phonon coupling constant, and $z$ is the direction normal to the surface.

To explore the microscopic dynamics of a surface exposed to laser pulse irradiation we modify the above continuum formulation, in conjunction with a classical MD simulation of the ionic dynamics [21]. The system is divided in the $z$ direction into two regions: a dynamic region (D) $0<z \leqslant z_{d}$ (with the origin taken at the topmost crystalline layer) and a continuum region (C) for $z_{\mathrm{d}}<z \leqslant z_{\mathrm{b}}$, where $z_{\mathrm{b}}$ is a position deep in the material. The ionic system in region $D$ is set up in the desired crystallographic structure (with $n_{\mathrm{d}}$ dynamic layers, positioned on top of $n_{\mathrm{s}}$ static layers) in a calculational cell which is periodically replicated in the two directions parallel to the surface, and the equations of motion for the ions, interacting via EAM potentials, are integrated in this region (except for the bottom static substrate) using the 5th-order Gear predictor-corrector algorithm [30] with a time step $\Delta t=5.1 \mathrm{fs}$. In our simulations we use for the (110) surface $n_{\mathrm{d}}=60$, $n_{\mathrm{s}}=4$, and for the (111) surface $n_{\mathrm{d}}=51, n_{\mathrm{s}}=3$ (this corresponds to 88 and 100 atoms per layer, for the (110) and (111) systems, respectively, and the thickness of region $D$ is $\sim 100 \AA$ ).

The energy exchange between the electron fluid subsystem and the dynamic ions is implemented by introducing an additional term into the ionic equations of motion

$M \frac{\mathrm{d}^{2} \boldsymbol{R}_{i}}{\mathrm{~d} t^{2}}=-\nabla_{\boldsymbol{R}_{i}} E_{\mathrm{EAM}}\left(\left\{\boldsymbol{R}_{i}\right\}\right)-\mu M \frac{\mathrm{d} \boldsymbol{R}_{i}}{\mathrm{~d} t}$,

where $M$ and $\boldsymbol{R}_{i}$ are the mass and position vector of ion $i$. The first term on the right is the EAM force on the $i$ th ion, and the coupling coefficient in the energy exchange term is given by [31a] $\mu=\mu(z, t)$ $=\left(G / C_{1}\right)\left\langle\left\langle T_{1}(z, t)\right\rangle-T_{\mathrm{e}}(z, t)\right] /\left\langle T_{1}(z, t)\right\rangle$. The electronic temperature $T_{\mathrm{e}}(z, t)$ over the whole region $z \leqslant z_{\mathrm{b}}$ is obtained via solution of Eq. (1) between successive MD steps (using a Runge-Kutta-type second-order method [30], with a time step $0.8(\delta z)^{2} /$ $2 D_{\mathrm{e}}$, where $D_{\mathrm{e}}=\kappa_{\mathrm{e}} / C_{\mathrm{e}}$ is the thermal diffusivity of the electrons, and $\delta z$ corresponds to a length equal to 3 interlayer distances). To get the lattice tempera-

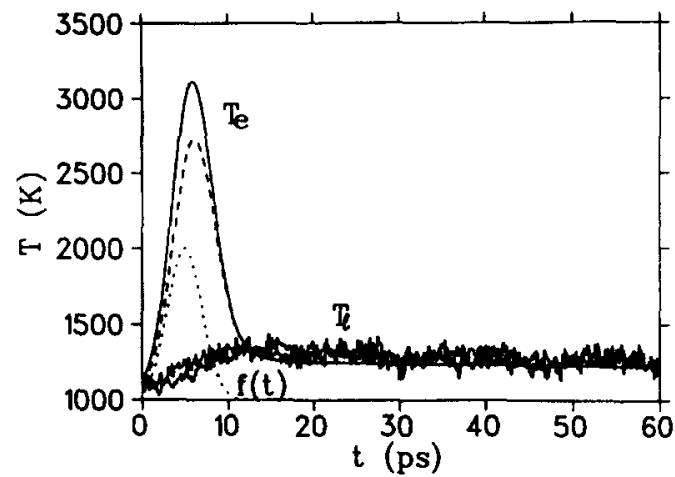

Fig. 1. Electronic temperature $\left(T_{\mathrm{e}}\right)$ at the surface region, consisting of the topmost two layers (solid), and deep in the dynamic region, near the static substrate (dashed), and the corresponding lattice (ionic) temperatures $\left(T_{\mathrm{l}}\right)$ obtained in a simulation using a superheating pulse $I_{0}=5 \times 10^{9} \mathrm{~W} / \mathrm{cm}^{2}$, starting from a $\mathrm{Cu}(111)$ surface equilibrated at $1100 \mathrm{~K}$. Also shown is the temporal shape of the laser pulse (dotted line). Temperature and time in units of $K$ and ps.

ture profile $T_{1}(z, t) \mathrm{Eq}$. (2) (with the first term neglected) is solved for the region $z_{\mathrm{d}} \leqslant z \leqslant z_{\mathrm{b}}$. For the dynamic region $0<z<z_{d}, T_{1}=\left\langle T_{1}(z, t)\right\rangle$ is obtained from the particle kinetic energies by averaging the simulation trajectories over $25 \Delta t$ intervals in regions of thickness $\delta z$. The boundary conditions used in solving these equations are: $T_{\mathrm{e}}\left(z_{\mathrm{b}}, t\right)=$ $T_{1}\left(z_{\mathrm{b}}, t\right)=T_{\mathrm{b}} \quad\left(z_{\mathrm{b}}=5000 \AA\right.$ and $\left.T_{\mathrm{b}}=1100 \mathrm{~K}\right)$; $\mathrm{d} T_{\mathrm{e}}(0, t) / \mathrm{d} z=0$ and $\mathrm{d} T_{\mathrm{e}}\left(z_{\mathrm{b}}, t\right) / \mathrm{d} z=$ $\mathrm{d} T_{1}\left(z_{\mathrm{b}}, t\right) / \mathrm{d} z=0$. The material and pulse parameters which we use are: $C_{\mathrm{e}}=96.6 T_{\mathrm{e}} \mathrm{J} / \mathrm{m}^{3} \cdot \mathrm{K}[32 \mathrm{a}]$, $C_{1}=3.5 \times 10^{6} \mathrm{~J} / \mathrm{m}^{3} \cdot \mathrm{K}[32 \mathrm{a}], \kappa_{\mathrm{e}}=166 \mathrm{~W} / \mathrm{m} \cdot \mathrm{K}$ $[31 \mathrm{~b}, 32 \mathrm{a}], G=1 \times 10^{17} \mathrm{~W} / \mathrm{m}^{3} \cdot \mathrm{K}[22], R=0.8$ and $\alpha=5 \times 10^{8} \mathrm{~m}^{-1}[32 \mathrm{~b}]$.

Typical records of the electronic and lattice temperatures in our simulations are shown in Fig. 1 where we exhibit results obtained for a laser pulse, with $I_{0}=5 \times 10^{9} \mathrm{~W} / \mathrm{cm}^{2}$ (leading to superheating of the surface, see below), irradiating a $\mathrm{Cu}(111)$ surface, equilibrated initially at $1100 \mathrm{~K}$. We observe significant heating of the electronic subsystem near the surface, as well as in a deeper region (though to a somewhat lower temperature, achieving a peak value slightly after the surface region), and a transfer of energy to the ionic degrees of freedom, whose temperature comes close (slightly above) to that of the electron fluid shortly after the termination of the laser pulse, equilibrating to $T_{\mathrm{e}}$ at longer times [33]. 
The temporal and spatial evolution of order and energetics for $\mathrm{Cu}(111)$ and $\mathrm{Cu}(110)$ are given in Figs. 2 and 3. Comparison of the order parameters for the (111) and (110) surfaces (Figs. 2a and $2 c$, respectively) irradiated by the same laser pulse $\left(I_{0}=\right.$ $\left.5 \times 10^{9} \mathrm{~W} / \mathrm{cm}^{2}\right)$ shows that while the $(110)$ surface (as well as the (100) surface, not shown) underwent melting induced by the laser heating, crystalline order is maintained at the (111) surface which remains superheated for $\sim 40 \mathrm{ps}$, with the lattice temperature rising as much as $40 \mathrm{~K}$ over the calculated bulk melting point $\left(T_{\mathrm{m}}^{\mathrm{b}}=1284 \mathrm{~K}\right.$ [26]). We further observe that irradiation by a higher-intensity pulse $\left(I_{0}=8 \times 10^{9} \mathrm{~W} / \mathrm{cm}^{2}\right)$, does result in melting of the (111) surface (see Fig. 2b). It is interesting to note that the disordering and melting of $\mathrm{Cu}(110)$ occurs simultaneously for a broader surface region (Fig. 2c), compared to the layer-by-layer process at the $\mathrm{Cu}(111)$ surface (Fig. 2b).

Having observed superheating of a perfect (111) surface it is instructive, for experimental as well as theoretical considerations, to inquire about the possible influence of defects on the ability to sustain a superheated state of a solid surface. Since surface melting has been observed to be initiated by generation of surface vacancies, we have performed simulations of laser irradiation of defective surfaces. Surprisingly, we observed superheating of $\mathrm{Cu}(111)$ surfaces even for highly defective surfaces (e.g., imperfect surfaces with up to $10 \%$ preexisting vacancies, either randomly distributed, or in a vacancy cluster). Moreover, laser irradiation of a $\mathrm{Cu}(111)$ surface which was equilibrated with a preexisting vacancy cluster (vc) and an adlayer island (see Fig. 3 (top)), resulted in superheating coupled with annealing of the surface structure (see Fig. 3 (bottom)). The annealing process occurred via a non-diffusional mechanism. Rather, we observed local embedding (settling) of the adatoms into the underlying topmost surface layer inducing cooperative displacements in that layer and the layer below, resulting in contraction and eventual filling of the vacancy cluster (see Fig. 3 (bottom)). Such a non-diffusional settling mechanism was observed by us in the context of studies of stability and spreading mechanisms of metallic nanostructures supported on metal substrates (Refs. $[11,12]$ and Section 2.2).

The remarkable stability of $\mathrm{Cu}(111)$ (and the (111)

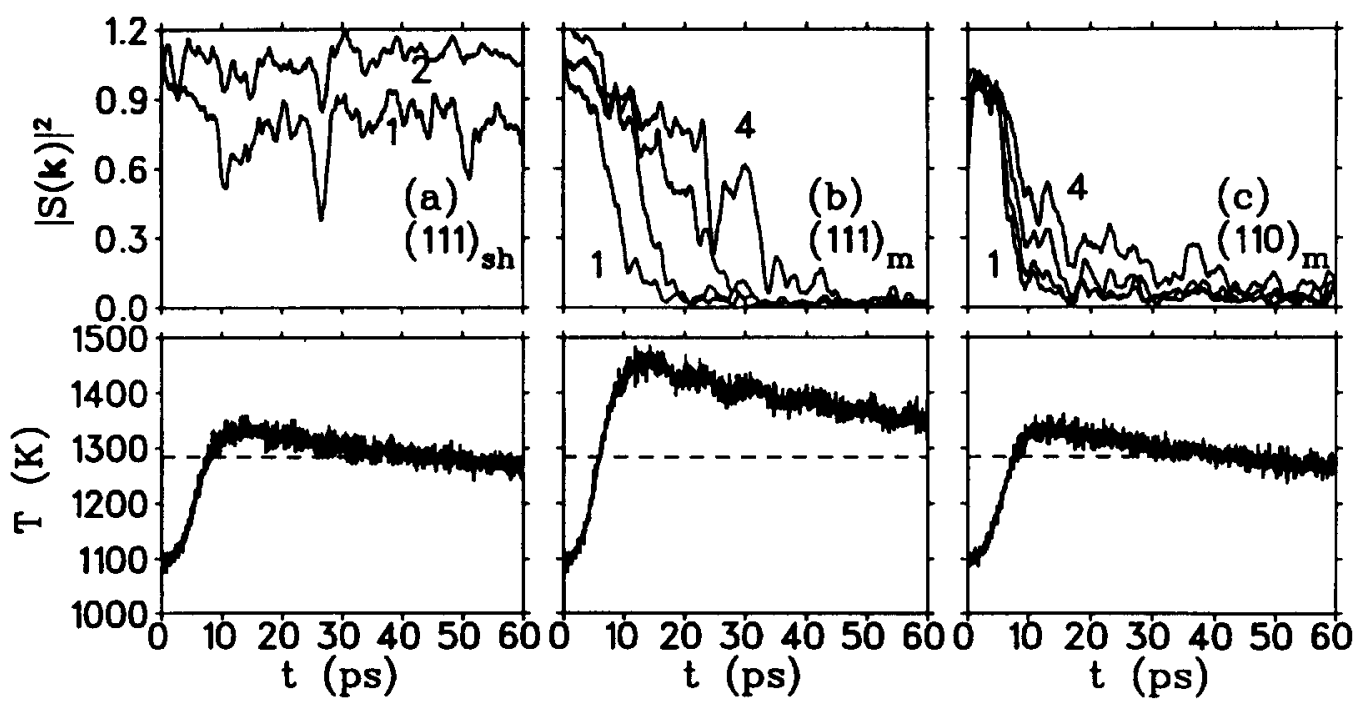

Fig. 2. Temporal evolution of the structure factor $|S(k)|^{2}$, for $k=4 \sqrt{2} \pi / a \sqrt{3}(0,1)$ for $\mathrm{Cu}(111)$ and $k=2 \pi / a(1,0)$ for $\mathrm{Cu}(110)$ and temperature of the whole dynamic system for laser irradiation MD simulations of: (a) superheating of the $\mathrm{Cu}(111)$ surface; (b) melting of the $\mathrm{Cu}(111)$ surface; (c) melting of the $\mathrm{Cu}(110)$ surface. In the $|S(k)|^{2}$ plots the structure factors for individual layers are denoted by layer numbers, with the topmost surface layer $l=1$. The calculated bulk melting temperature $\left(T_{\mathrm{m}}^{\mathrm{b}}=1284 \mathrm{~K}\right)$ is denoted by a dashed line in the temperature plots. $|S(k)|^{2}$ normalized to its value at the initial temperature $T=1100 \mathrm{~K} . T$ in $\mathrm{K}$ and time in ps. 
surfaces of other fcc metals) against melting correlates with the high energy for formation of vacancy-adatom pairs $\left(E_{\mathrm{vaf}}\right)$ at the surface. An adequate estimate of $E_{\text {vaf }}$ may be obtained by considering the change in coordination, $\Delta_{c}$, of an atom when it is moved from its original position in the surface to the adlayer (i.e., the net number of "broken bonds"). The relative stability of $\mathrm{Cu}(111)$ as compared to the $\mathrm{Cu}(110)$ surface is reflected in the values $\Delta_{\mathrm{c}}(111)=9-3=6$ and $\Delta_{\mathrm{c}}(110)=7-5=$ 2, which together with $E_{\text {vaf }}(110)=0.4 \mathrm{eV}$ [26] yield a lower-bound estimate of $E_{\mathrm{vaf}}(111)=1.2 \mathrm{eV}$. (We remark that in a bond-breaking model the cohesive energy of a given atom varies linearly with coordination number $(C)$. The many-atom interactions in EAM models result in a nonlinear dependence of
$E(C)$ which effectively increases the change in energy when going to low $C$ [34].) A similar argument applied to the (111) surface containing a vc shows that the energy cost for formation of a vacancyadatom pair from the periphery of the $\mathrm{vc}$ is $(2-2.5)$ $\times E_{\mathrm{vaf}}(110)$ for the concave edge of the $\mathrm{vc}$, and is larger than $E_{\mathrm{vaf}}(110)$ even for the convex edge of the adatom island (see Fig. 3). Consequently the stability of such clustered defects on the (111) surface may be attributed to a relatively high "line tension" which prohibits mobility and nucleation of surface melting.

The results of these MD simulations of laser pulse irradiation of copper surfaces, where coupling between the ionic and electronic degrees of freedom was incorporated, provide significant insights per-

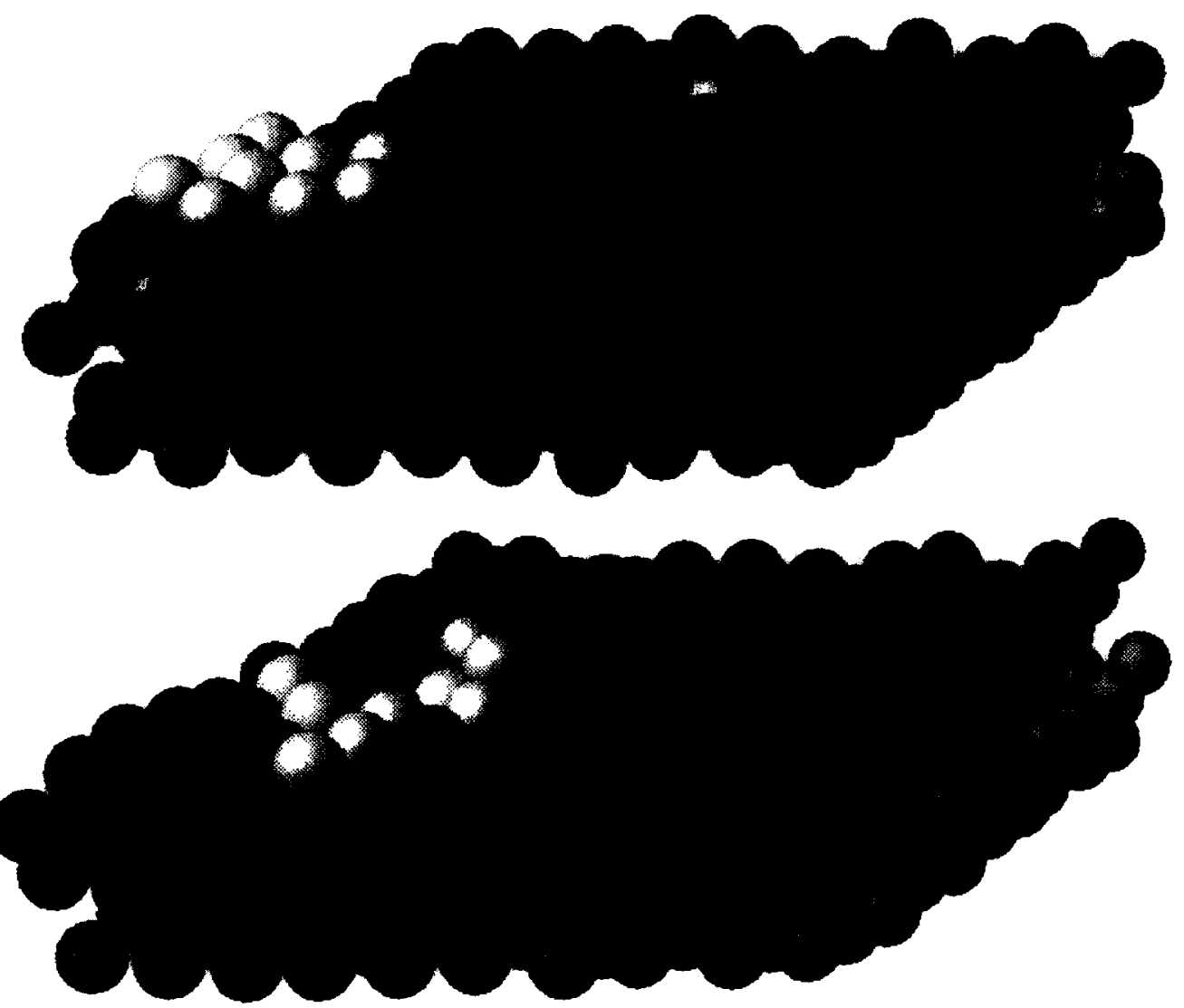

Fig. 3. Atomic configuration of a $\mathrm{Cu}(111)$ surface containing a vacancy cluster and an adlayer island (brightest atoms) prior to irradiation (top), and a configuration recorded $20 \mathrm{ps}$ after the termination of a superheating laser pulse (bottom). Dark and grey spheres correspond to atoms in the topmost surface layer and in the layers below it. Note that in the annealed surfaces the atoms originating at the adlayer island did not migrate to fill the vacancy cluster [10]. 
taining to dynamics, energetics, and structure of surface superheating and melting processes. Furthermore, the observations of superheating and structural annealing of the (111) surface suggest further experiments as well as surface processing techniques.

\subsection{Stability and collapse of metallic nanostructures on surfaces}

Basic and technological interest in the physical properties of small materials structures, and in lo-
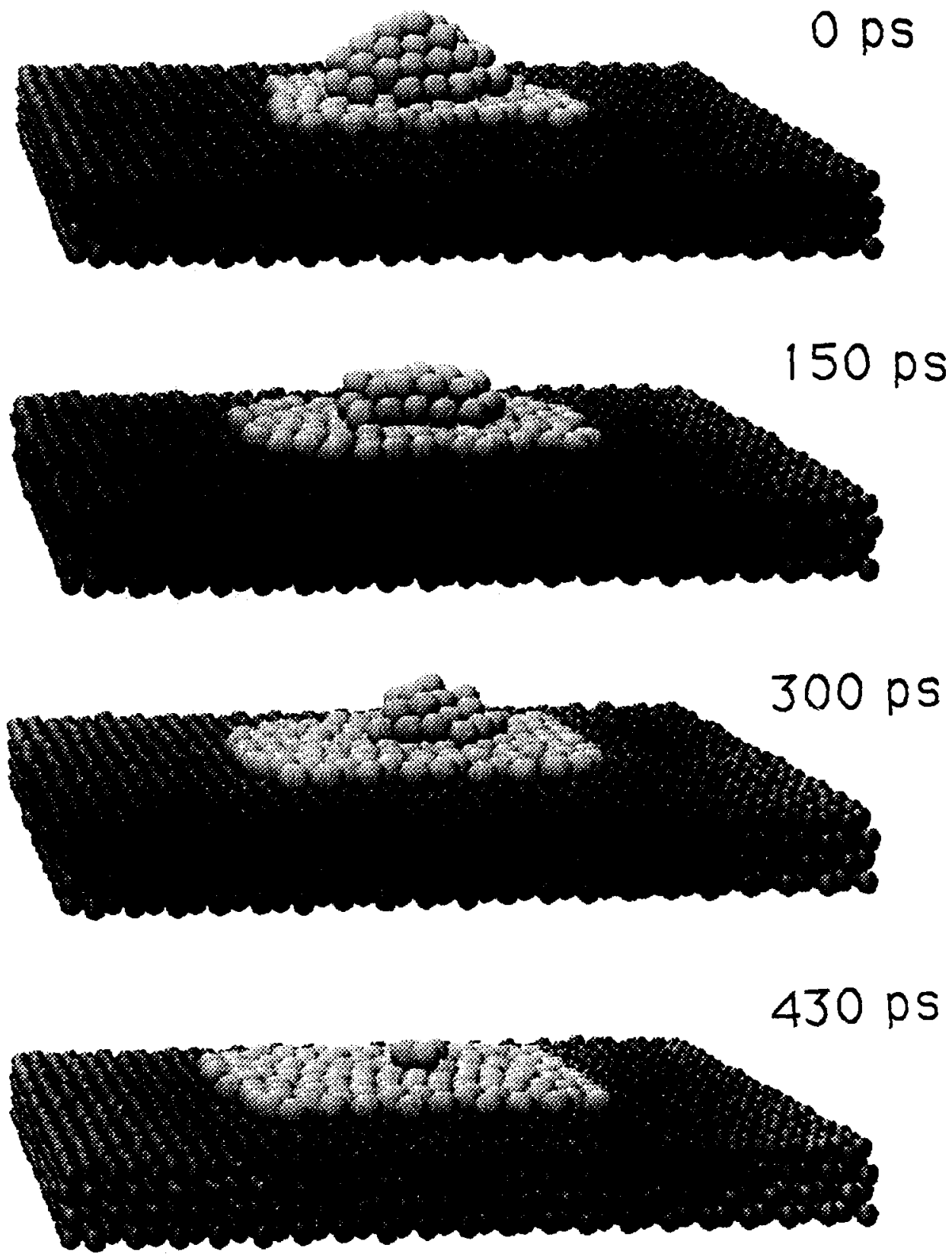

Fig. 4. Selected atomic configurations from simulations $[11,12]$ of the collapse of an Au cluster (light balls) on Ni(100) (dark balls) at $600 \mathrm{~K}$. 
cally controlled surface modifications, underlie intensifying research endeavors in this area [6,7]. Using molecular dynamics (MD) simulations, we have explored the structure, energetics, dynamics, stability and collapse mechanisms of surface-supported metallic nanostructures which are key issues in preparation, control, and exploitation of such structures.

In the MD simulations $[11,12]$ we employed the EAM potentials [36], for systems consisting of solid substrates containing $N_{\mathrm{d}}$ layers of dynamic atoms, exposing $\mathrm{Ni}(100)$ or $\mathrm{Au}(111)$ surfaces, with $N_{\mathrm{L}}$ atoms per layer. These dynamic substrates were positioned on top of $N_{\mathrm{s}}$ static layers with the corresponding geometry, and temperature was controlled via scaling of particle velocities at the bottom layer of the dynamic substrate. Periodic boundary conditions were imposed in directions parallel to the surface plane. Prior to simulations of the collapse processes, these systems, with deposited 3D gold clusters, were equilibrated at room temperature $(300 \mathrm{~K})$.

In earlier simulations (Refs. [13,14], and Section 2.3) we have shown that contact between a metallic tip and a substrate (e.g., $\mathrm{Ni}$ or $\mathrm{Au}$ tips and a $\mathrm{Au}$ surface) and subsequent slow retraction of the tip generates an elongated junction made of the softer metal $(\mathrm{Au})$. Upon breaking of the contact a mound of gold is left on the surface. Moreover, the size and height of the mound increase when the retraction follows a slight indentation of the surface $[13,14]$. Indeed, gold mound formation via such a process has been demonstrated in recent STM experiments (see Pascual et al., in Ref. [35]). The 3D Au nanostructures which we investigated below were produced in this manner. We also mention recent investigations suggesting that metallic clusters containing a few hundred atoms can be "soft-landed" on surfaces to form 3D deposited structures [37].

Our simulations show that heterostructures, such as a three-dimensional (3D) crystalline gold cluster (dot) deposited on a $\mathrm{Ni}(100)$ surface, where the lattice mismatch is large $(16 \%)$ and the surface energy of the nickel substrate is larger than that of the deposited Au dot, can collapse via a non-diffusional solid-on-solid settling (SOSS), mechanism. The SOSS involves a succession of layer-by-layer incorporations (embedment) of $\mathrm{Au}$ atoms from a given layer into the underlying Au layer, accompanied by subsequent cooperative stress relief via lat- eral displacements of atoms in the underlayer. These processes result ultimately in transformation of the initial 3D structure into a 2D adsorbed gold island whose area grows linearly with time $[11,12]$. On the other hand a homostructure, e.g. a 3D crystalline gold cluster deposited on an $\mathrm{Au}(111)$ surface, adsorbs epitaxially (in registry) with the substrate, exhibits enhanced stability and collapses at elevated temperatures via a dislocation mediated settling (DMS) mechanism, involving vacancies generated by surface dislocations in the deposited $\mathrm{Au}$ cluster. These novel atomic-scale mass transport and collapse mechanisms and the interplay between interfacial energetics and structure which they portray, pertain to a number of interfacial processes (e.g., deposited nanostructures, thin film growth, annealing of damage, surface manipulations and nano-scale lithography).

$A u$ dot on a Ni(100) surface. A sequence of atomic configurations illustrating collapse at $600 \mathrm{~K}$ of a 3D Au dot containing 154 atoms (with initially $n_{1}=97, n_{2}=29, n_{3}=17$, and $n_{4}=11$ in each of the layers of the deposited cluster; $N_{\mathrm{d}}=4, N_{\mathrm{L}}=800$ and $N_{\mathrm{s}}=3$ ) is shown in Fig. 4 and the corresponding kinetics of the process is described in Fig. 5. The core of the interfacial $\mathrm{Au}$ layer (layer 1 of the $\mathrm{Au}$ cluster), forms a (111) structure rotated by $30^{\circ}$ with respect to the underlying $\mathrm{Ni}(100)$ surface (driven by the large lattice constant mismatch between the two materials) and the structure close to the periphery of the interfacial layer is less ordered. The areal spreading of the $\mathrm{Au}$ interfacial layer is linear in time (Fig. 5a). Furthermore, the radial atomic distributions in the resulting 2D Au island shown in Fig. 5b illustrate that atoms nearer to the middle of the island originated from higher layers in the 3D Au structure (that is layers more distant from the $\mathrm{Ni}(100)$ surface) while those closer to the periphery of the final 2D island correspond to atoms located initially in the interfacial layer of the Au cluster. This atomic distribution is inverted from the one that would have resulted if spreading occurred via diffusional transport and attachment of atoms from higher layers to the periphery of the interfacial, bottom, layer. Rather, it is a consequence of the SOSS mechanism where atoms at the periphery of layer $l+1$ in the 3D Au cluster drop (embed) into the underlying $l$ th layer inducing lateral radial outward atomic displacements 

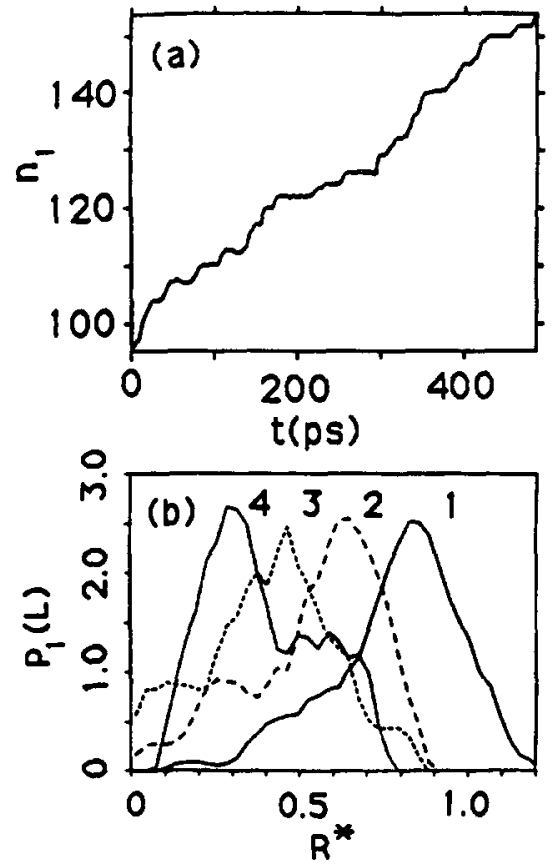

Fig. 5. (a) Number of particles $\left(n_{1}\right)$ in the interfacial (bottom) layer of the adsorbed Au cluster (see Fig. 4), plotted versus time. (b) Radial distributions, $P_{1}(L)$, of $A u$ atoms in the interfacial layer (i.e., the 2D Au island at the end of the collapse process, see bottom of Fig. 1), distinguished according to the layer of the cluster $(1 \leqslant L \leqslant 4)$ from which they originated, plotted versus $R^{*}$, with the origin at the center of mass of the 2D patch. $R^{*}$ is the distance of atoms from the origin, normalized by the mean radius of the island. $P_{1}(L)$ is normalized to unity for each $L$.

in that layer. Such embedment sequences, driven by the gain in cohesive energy due to the interaction between $\mathrm{Au}$ and $\mathrm{Ni}$ atoms propagate from the top layer of the settling Au cluster downward, culminating in lateral spreading of the bottom (interfacial) $\mathrm{Au}$ layer on the $\mathrm{Ni}(100)$ surface.

$A u$ dot on an $A u(111)$ surface. We have verified first that our simulations reproduce the $23 \times \sqrt{3}$ surface reconstruction observed on $\mathrm{Au}(111)$ [38], consisting of densification of the topmost layer via formation of a series of discommensurations, separating regions with fcc and faulted hcp stacking sequences, along the [110] direction. Furthermore, we have observed that the presence of an adsorbed island causes local dereconstruction of the underlying gold layer.

Simulations of an Au cluster, similar to that described before, adsorbed epitaxially (in registry) on an $A u(111)$ surface, have shown that while in the initial stages of the collapse embedding processes of similar nature to those discussed above take place, the process slowed down progressively and continuation of the collapse (at least on time scales accessible to $\mathrm{MD}$ simulations) required heating to $T \gtrsim 800 \mathrm{~K}$. At this later stage a cross-over to a dislocation mediated mechanism occurred (the aforementioned DMS), associated with shrinking of the areas of the topmost layers of the collapsing cluster and consequent increases in the distances between the peripheries of successive layers. These processes result in larger barriers for embedment (see below) thus suppressing the direct embedding mechanism. During the evolution of this system the DMS mechanism became particularly evident at the stage when the collapsing cluster consisted of two layers, an interfacial one and a small island on top; direct embedding occurred only when the top island migrated close to the periphery of the interfacial layer (see below).

To further investigate the DMS mechanism we show in Fig. 6a the structure of a two-layer $\mathrm{Au}$ cluster consisting initially of a bottom interfacial layer and a top island containing 505 and 54 atoms, respectively, adsorbed and initially equilibrated at $300 \mathrm{~K}$ and then heated to $900 \mathrm{~K}$, on an $\mathrm{Au}(111)$ substrate with $N_{\mathrm{d}}=2$ and $N_{\mathrm{L}}=1216$, together with atomic configurations illustrating structural evolution during a 30 ps interval (Figs. 6b, 6d). Also included are fault contours (Fig. 6c corresponding to the structure in Fig. 6b), representing the displacements of atoms in the interfacial layer of the deposited cluster from their perfect fcc lattice positions. These contours illustrate reconstructed regions of fcc (C) and hcp (A) stacking (note that the hcp and discommensuration region on the left of the island indicate densification mainly along the [1ㅣㅣ direction, and the ones on the right are along the [1 $\overline{2} 1]$ direction; short time scale dynamical fluctuations in the degree of perfection of the reconstruction patterns were observed throughout our simulations of finite gold islands at elevated temperatures).

Starting from the Au cluster shown in Fig. 6a the structural evolution shown in Figs. 6b, 6d involves generation of slip along the [101] direction and an array of partial dislocations [39] (Fig. 6b) accompanied by formation of surface partial dislocations (Fig. $6 \mathrm{~d})$ with component Burgers vectors in the $[1 \overline{2}]$ and 


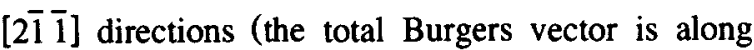
$[10 \overline{1}])$. These dislocations are confined to the plane of the interfacial layer of the gold cluster [40], and
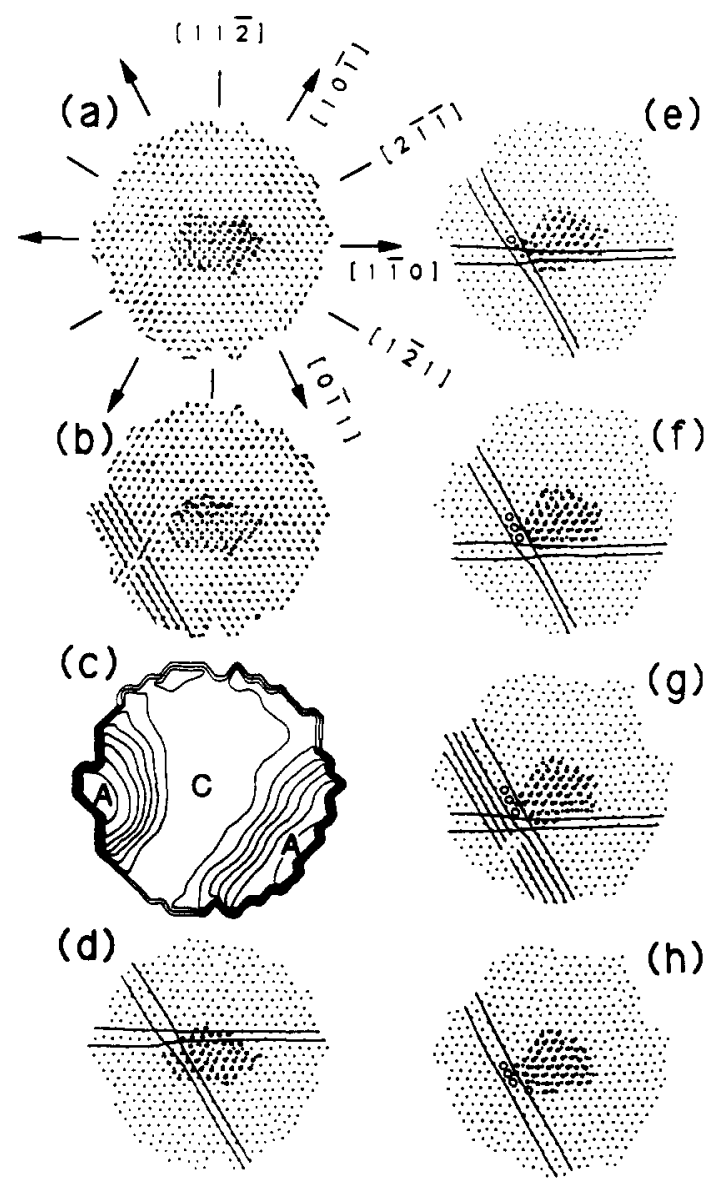

Fig. 6. Top views of atomic configurations (and short-time trajectories, in (a) and (b)) selected from simulations of a two-layer Au cluster adsorbed on an Au(111) surface, illustrating dislocation mediated settling (DMS). Only atoms of the adsorbed cluster are shown. The initial structure of the Au cluster, consisting of an interfacial layer (which exhibits an Au(111) reconstructed structure) and a second-layer island, is shown in (a). Formation of slip along the $[10 \overline{1}]$ direction is shown in (b), along with corresponding fault contours in (c) ( $A$ and $C$ denote hcp and fcc regions, respectively, separated by discommensuration regions. Mean displacements from perfect fcc positions are $0.4 \AA$ in $C$ and $1.5 \AA$ in $A$, and the increment between contour lines is $0.15 \AA$ ). Surface dislocations intersecting at the periphery of the second-layer island and formation of a vacancy are shown in (d), followed by a later configuration (e) where an embedded atom from the second layer filling the vacancy is indicated by an empty circle. Subsequent evolution leading to settling of three additional atoms into the interfacial layer is shown in (f)-(h). Thin guiding lines are drawn in (b) and (d)-(h). get pinned at the periphery of the second-layer island leading to formation of a vacancy in the interfacial layer (Fig. 6d). Subsequently the vacancy gets filled by an atom originating from the second-layer island (see Fig. 6e where the embedded atom is marked by an empty circle).

Further evolution of the system, 260 ps later, during a 15 ps interval, illustrating dislocation mediated settling of three additional second-layer atoms, is shown in Figs. $6 \mathrm{e}-6 \mathrm{~h}$. These atomic configurations illustrate movement of the dislocations (compare Figs. 6e and 6d), and climb of the dislocations via incorporation of two second-layer atoms (Fig. 6f)). Initiation of further slip along the [101] direction and formation of surface partial dislocations are shown in Fig. 6g. These processes resulted in annihilation of the dislocations leaving a vacancy in the interfacial layer. Finally the vacancy was filled by an atom displaced into it via settling of an additional atom from the top layer of the cluster (Fig. 6h).

The embedding energetics and structural properties of layers of the adsorbed Au clusters which are distant from the supporting substrate are similar in the two cases (i.e., on the $\mathrm{Ni}(100)$ and $\mathrm{Au}(111)$ substrates), while they differ markedly in the interfacial region, proximal to the surface, which governs the spreading propensity and rate. This is largely due to the difference in the surface energies of the two materials. Calculations show [11] that the energy barrier for lateral detachment of an atom from the second-layer island of a two-layer gold cluster adsorbed on $\mathrm{Au}(111)$ is $\sim 0.9 \mathrm{eV}$ and further displacements along the surface require a smaller diffusion barrier $(0.13 \mathrm{eV})$, with an increased barrier $(0.16 \mathrm{eV})$ for "falling over the edge" to the underlying adsorbed layer (such increased barriers for migration over descending step edges have been discussed recently in the context of surface diffusion and thin film growth modes [41]). Furthermore, embedment of an atom near the edge of the second-layer island involves a barrier of $0.8 \mathrm{eV}$ and is accompanied by spontaneous promotion of a neighboring atom from the interfacial layer of the structure to the layer above it, thus resulting in no net change in the number of atoms in these layers. Consequently, diffusion and direct embeddment processes are greatly suppressed for the homostructure system.

Similar calculations for the same Au cluster but 
adsorbed on a $\mathrm{Ni}(100)$ surface have shown that detachment of an $\mathrm{Au}$ atom to a distance of $\sim 1 \AA$ from the periphery of the second-layer island (involving a barrier of $\sim 0.5 \mathrm{eV}$ ), is accompanied by a spontaneous incorporation into the interfacial layer leading to spreading.

From these results we conclude that the collapse of a heterostructure, such as a 3D Au cluster adsorbed on $\mathrm{Ni}(100)$ can occur via a settling mechanism (SOSS), involving local atomic incorporation (embedment into underlayers). These processes are driven by the resulting gain in interfacial cohesion and are aided by the large lattice mismatch between the two materials, which reduces the interfacial frictional resistance to stress relief via outward lateral atomic displacements, resulting in spreading. On the other hand for Au clusters adsorbed on Au(111) the much reduced energetic driving force, coupled with high interfacial friction due to the epitaxial structure of the interface between the deposited cluster and the substrate, result in higher stability compared to that of $\mathrm{Au} / \mathrm{Ni}(100)$. In this case collapse of the deposited structure at elevated temperatures is mediated by a settling mechanism involving spontaneous generation and propagation of surface dislocations (DMS). While diffusion has been invoked in interpretation of experimental results pertaining to collapse of nanostructures on surface, no direct evidence for diffusion events was given [35]; this does not preclude diffusion to the edge of deposited single atoms, where there is no detachment barrier, or of very small clusters, as observed in FIM experiments [41a]. The alternative collapse mechanisms which we found may dominate in certain ranges of sizes and temperatures in the systems studied by us as well as in other materials (see Ref. [10], and the previous section).

As we noted above, together with the collapse processes, the top layer of the gold cluster deposited on $\mathrm{Au}(111)$ (see Fig. 6) migrated, from its initial location at the center, towards the periphery of the underlying layer of the two-layer cluster. Such migration and diffusion processes are important in the growth of thin films via deposition of atoms or clusters, and have been studied experimentally [41$44]$, and theoretically [45-49].

The most common descriptions of the diffusion of islands on surfaces invoke periphery diffusion or evaporation-condensation mechanisms. In contrast

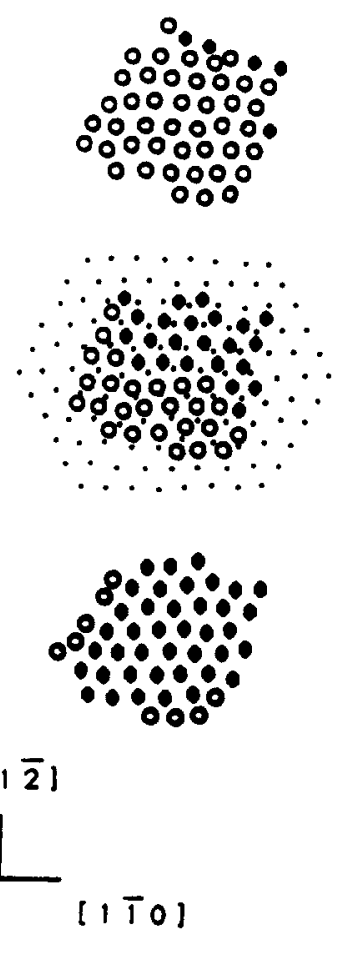

Fig. 7. Atomic configurations of the top-layer island of a two-layer cluster deposited on Au(111), illustrating the misfit dislocation mechanism for diffusion, recorded in a MD simulation at $900 \mathrm{~K}$. At the top and bottom only the atoms of the top-layer island are shown, and in the middle the small dots denote the positions of gold atoms of the underlying layer in the vicinity of the island. Open circles denote hcp adsorption sites of the underlying Au(111) layer, and filled circles correspond to fcc sites. The net translation of the island during this 6 ps event is by $a / \sqrt{3}$ in the $[\overline{2} 11]$ direction, and involves generation and propagation of a misfit dislocation (2D stacking fault). Note the intermediate configuration (middle) consisting of fcc and hep domains.

to these single particle mechanisms, we have observed in our simulations that while a certain degree of atomic diffusion around the island periphery has occurred, the dominant mechanism of the top island migration is cooperative in nature, involving generation and propagation of a misfit dislocation (which may also be described as a stacking fault) in the migrating island. This novel cooperative diffusion mechanism is illustrated in Fig. 7, where three configurations of the island, resulting in net translation of the island by $a / \sqrt{3}$ ( $a$ is the nearest neighbor distance) in the [ $\overline{2} 11]$ direction, are shown. In the initial state (Fig. 7 (top)) the large majority of the 
island atoms occupy hcp sites on the (111) surface (recall that an adsorbed island leads to local dereconstruction). The middle configuration in Fig. 7, recorded 3 ps later in the simulation, exhibits an island with two domains (hcp and fcc) with a two-dimensional stacking fault (or misfit dislocation) between them. Three ps later (Fig. 7, (bottom)) the island transformed, via migration of the misfit dislocation line, to one where the majority of atoms occupy fcc sites, resulting in a net translation of the island. All the atomic motions occurring in the above migration processes are across bridge sites of the (111) surface net, which are the energetically preferred paths. Consequently, all the island diffusion events occur along the [2i $\overline{1}$ ] family of directions (see Fig. 6). Each of the cooperative island migration events described above occur rather rapidly (of the order of several picoseconds), and they can occur in succession to one another, or intermittently.

\subsection{Interfacial junctions and nanowires}

Tribological phenomena, which include friction, lubrication and wear processes, present immense theoretical, as well as experimental, challenges. Compounding the difficulties of studying such problems are the complex nature of the pertinent materials systems and their interactions (e.g., metals, ionics, semiconductors, oxides, ceramics, organic molecules, and polymers) and that of the interphase interfaces involved, in juxtaposition with the need to explore energetics, structure, dynamics, and transport processes in nonuniform systems under nonequilibrium conditions and beyond the linear response regimes for mechanical and flow characteristics. Consequently, until rather recently, most theoretical approaches to the aforementioned problems have been anchored in continuum formulations, such as continuum elasticity, contact mechanics, hydrodynamics, viscous flow, and elasto-hydrodynamics [50].

Investigations which combine advances in theoretical understanding of the nature of interatomic interactions in materials with the development of methods for computer-based materials modeling, open new avenues in our quest to explore the properties of materials with refined spatial and temporal resolution $[8,18]$. Such studies, performed for several materials systems characterized by different interatomic inter- actions and nature of bonding, have provided significant insights about the atomistic mechanisms underlying and governing the interfacial processes which occur when material bodies are brought into contact, are separated, or slide with respect to each other. Some of the results correlate well with the predictions of theories constructed on the basis of macroscopic considerations $[17,50]$. Thus these results provide a microscopic foundation for a rigorous derivation of such descriptions.

On the other hand, certain of the findings exhibit physical behavior which is unique to the nanorealm (i.e., to systems of very small characteristic dimensions). Such findings include atomic-scale adhesion and interfacial wetting phenomena occurring upon contact formation [13,14], generation of atomic-scale connective wires which develop upon separation of small intermetallic contacts $[13,14]$, atomic-scale stick-slip friction occurring upon sliding [52], and structural ordering and phase transitions occurring in thin lubricant films pressed and sheared between confining solid plates $[15,18,50 c, 53,54]$. These observations are of importance to the development of a fundamental understanding of the physical nature of materials of very small dimensions, assessment of the range of validity of macroscopic descriptions of the mechanical, rheological and flow properties of materials, and interpretation of experiments (particularly scanning tip-based microscopies, and the surface force apparatus). Moreover, understanding of the novel behavior of materials of very small dimensions is of relevance for development of nano and micro fabricated devices and atomic-scale structural manipulations of materials $[7,11]$.

\subsubsection{Solid junctions}

Ideas pertaining to the key issue in friction, namely understanding of the origins and nature of excitations and energy transfer and dissipation occurring when sliding two bodies in contact, date back several centuries [55]. Following a rather convolved and curious path, where the main theme centered on interlocking of morphological (geometrical) irregularities, the current view, which emerged over four decades ago, is that in the case of metals, and certain other materials, the friction force is essentially the force required to shear intermetallic junctions formed at the region of real contact between the interfacing 
materials bodies, plus the force required to plow the surface of the softer material by the asperities of the harder surface [56].

For metallic systems, consisting of a clean gold substrate and a nickel tapered and faceted tip (and for the reverse situation, i.e., a nickel surface and a gold tip), simulations revealed [13] the onset of an instability as the tip approaches the sample to a distance of $\sim 4 \AA$, causing a jump-to-contact (with gold atoms displacing about $2 \AA$ in about 1 ps) and formation of adhesive bonding between the two materials, driven and accompanied by atomic-scale wetting of nickel by gold atoms, due to differences in their surface energies. Furthermore, it was found that detraction of the tip from the surface after contact, results in a significant inelastic deformation of the sample exhibiting ductile extension, formation of a connective neck of atomic dimensions and eventual tear, yielding upon separation a gold coated nickel tip and a damaged gold surface.
These phenomena, which were observed also in simulations involving indentations of the substrate (i.e., penetration of the tip into the surface), exhibiting transitions from elastic to plastic mechanical response of metals and formation of extended atomically thin crystalline connective wires, are portrayed by marked hysteresis in the calculated force-versusdistance relation between the interacting materials bodies upon lowering and lifting of the tip from the surface (i.e., the force records do not track each other for the two processes). Furthermore, the simulations revealed the nature of the elongation process of such ordered crystalline atomic-scale junctions, consisting of straining and yielding stages occurring with a periodicity of the interlayer spacing in the wire, and being reflected in periodic oscillations in the recorded force (see Figs. 8 and 9, recorded in a simulation [13] of a nickel tip slightly indenting an $\mathrm{Au}(001)$ surface, and subsequently retracted from it). In each elongation stage, atoms in layers (mainly in

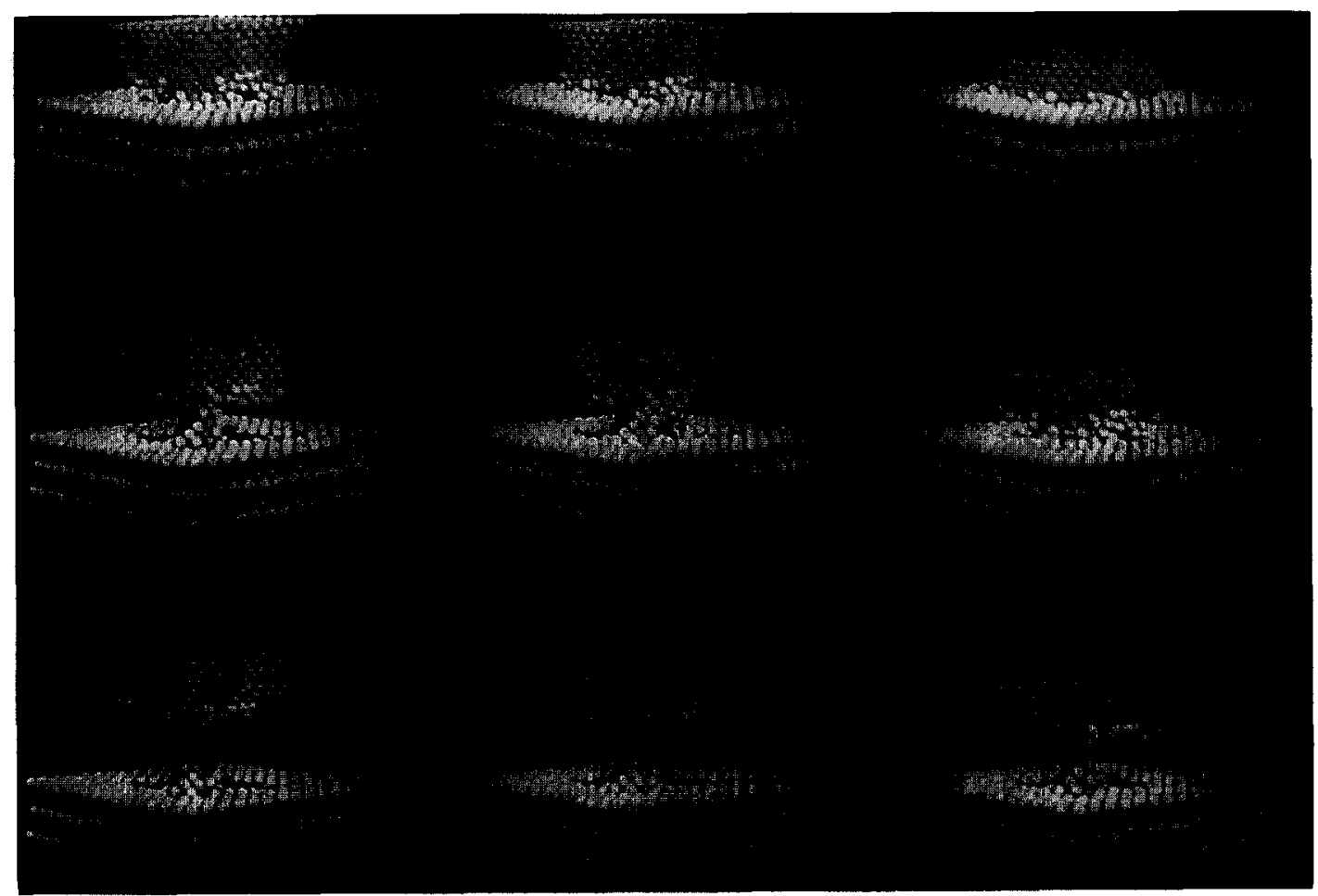

Fig. 8. Sequence of atomic configuration starting from a $\mathrm{Ni}$ tip indented in a $\mathrm{Au}(001)$ substrate (top right) and during the process of retraction of the tip (from right to left) accompanied by formation of a connective solid gold junction, and culminating in a junction of atomic dimensions (bottom left). In each frame the upper block represents the nickel tip, and the slab below (including the junction) represents the gold surface [13]. 
the vicinity of the narrowest part of the junction) respond first via accumulations of stress accompanied by the occurrence of strained configurations of the wire (which remains ordered in atomic layers, though with increasing nonuniformity of the interlayer spacings). This stage is followed by a shorter atomic disordering and rearrangement period culminating in the formation of an added layer, with a relief of the accumulated stress and restoration of a higher degree of order in the wire. Consequently, each such elongation-necking stage results in a more extended crystalline junction (in increments of the order of the interlayer spacing in the junction, i.e., $\sim 2 \AA$ ) of a smaller cross-sectional neck area. From these simulations it has been predicted [13] that gold nanowires are characterized by a critical yield stress of $\sim 2-3 \mathrm{GPa}$, i.e. an order of magnitude larger than that of bulk $\mathrm{Au}$, and of the order of the theoretical value calculated without dislocations.

To illustrate the elongation process we show in Fig. 10a side views of atomic configurations, starting from a layer-ordered junction containing 4 atomic layers (see the corresponding intra-layer atomic ar-

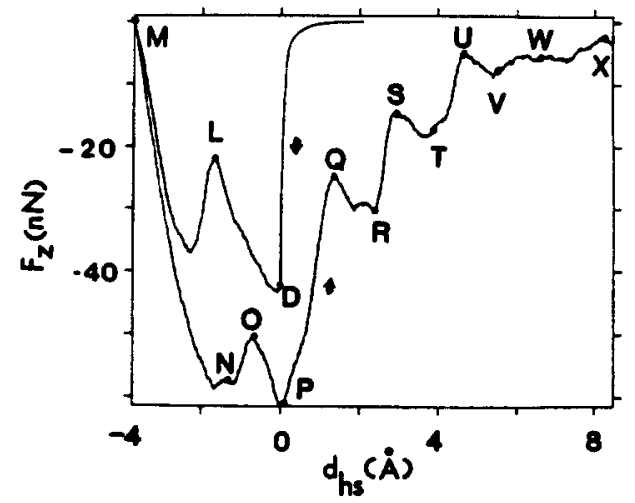

Fig. 9. Force on the tip atoms, $F_{z}$, versus tip-to-sample distance, reconded in a MD simulation [13] of a Ni tip interacting with an Au(001) surface at $300 \mathrm{~K}$. The simulation describes the tip approach to the surface, jump-to-contact (point $D$ ), indentation (points $D, L, M$ ), and subsequent retraction (points $M, N, P, \ldots, X$ ) of the tip from the surface. Note the marked hysteresis in the force upon retraction, and the oscillatory variations during elongation of the solid junction. $d_{\mathrm{hs}}$ denotes the distance between the rigid tip-holder assembly and the substrate ( $d_{\mathrm{hs}}$ is normalized to zero at the jump-to-contact point, marked D). The capital letters denote the actual distances, $d_{\mathrm{ts}}$, between the bottom part of the Ni tip and the top of the Au surface; $D=3.8 \AA, L=2.4 \AA, M=0.8 \AA$, $\mathrm{N}=2.6 \AA, 0=3.0 \AA, \mathrm{P}=3.8 \AA . \mathrm{A}, \mathrm{Q}=5.4 \AA \mathrm{A}, \mathrm{R}=6.4 \AA, \mathrm{S}=7.0$ $\AA, T=7.7 \AA, U=9.1 \AA, V=9.6 \AA \mathrm{W}=10.5 \AA$, and $\mathrm{X}=12.8 \AA$. rangements shown in Fig. 10b) and ending with a longer layer-ordered junction containing 5 layers, along with a structure during the intervening disordered stage. Corresponding plots of the atomic density profiles along the normal axis $(Z)$ of the junction shown in Fig. 10c illustrate the atomic distributions in the initial and final ordered stages of the junction as well as during the straining and disordering stages of the transformation.

During elongation the wire evolves through atomic configurations with various degrees of order and disorder. Note that even at the ordered stages, which exhibit crystalline-like atomic layers along the axis of the junction, the shapes of the layers are rather irregular (see Fig. 10b), resulting in a solid wire with a surface roughness of a few $\AA$, comparable to the wavelength of the conduction electrons in such wires $(\sim 4 \AA)$. While such an aspect of disorder may not greatly affect the mechanical characteristics it can influence electronic transport processes.

These theoretical predictions pertaining to the mechanical properties as well as the suggestion that such nano-scale wires could be used for investigations of quantum effects in electronic transport in ultra-thin wires and single-atom contacts [13], have been verified by recent experimental studies using atomic force microscopy (AFM) for investigations of the mechanical properties of atomic-scale solid junctions [13,57], and STM measurements at room temperature of electronic conductance quantization in atomically thin gold wires of up to $50 \AA$ length $[19,58]$, and a transition to a localization regime in wires whose extension (longer than $100 \AA$ ) is larger than the localization length [19].

In the conductance experiments [19], contact between the tip and the substrate is produced either by applying a voltage pulse or by indentation [58], starting from typical STM tunnel conditions. Once the contact is produced as indicated by the electrical current flowing between the two electrodes, it was elongated by retracting the tip slowly $(\sim 1 \AA / s)$. Gold evaporation onto mica and $\mathrm{Au}(110)$ single crystals were used as samples, and Pt/Ir and gold tips were employed interchangably (the results are insensitive to the kind of tip used; most likely, the $\mathrm{Pt} / \mathrm{Ir}$ tip apex is covered with gold atoms once the tip touches the sample [13]). Since the wires are more easily formed in air, the data presented in this paper 
were measured under ambient conditions. However, similar results were obtained in UHV. Experiments were performed on a large number of wires (both short and long ones). Typical data are shown in Fig. 11 for the conductance of a short wire $(\sim 50 \AA)$ produced by bringing a Pt/Ir tip to contact with a gold surface at room temperature and subsequently elongating the contact slowly by retracting the tip (a constant bias voltage of $32 \mathrm{mV}$ was applied during the process). The voltage was kept constant during the measurements by using a low gain $I-V$ converter as explained in Ref. [58a].

The appearance of room-temperature quantized conductance in the short wires and its persistence (see Fig. 11 where 9 steps of height $2 e^{2} / h$ or $2\left(2 e^{2} / h\right)$, with a period $d \approx 2 \AA$, the interlayer spacing in the material, are seen) are quite remarkable, particularly in view of the known sensitivity of conductance quantization to the presence of disorder (which in our wires includes surface roughness, and
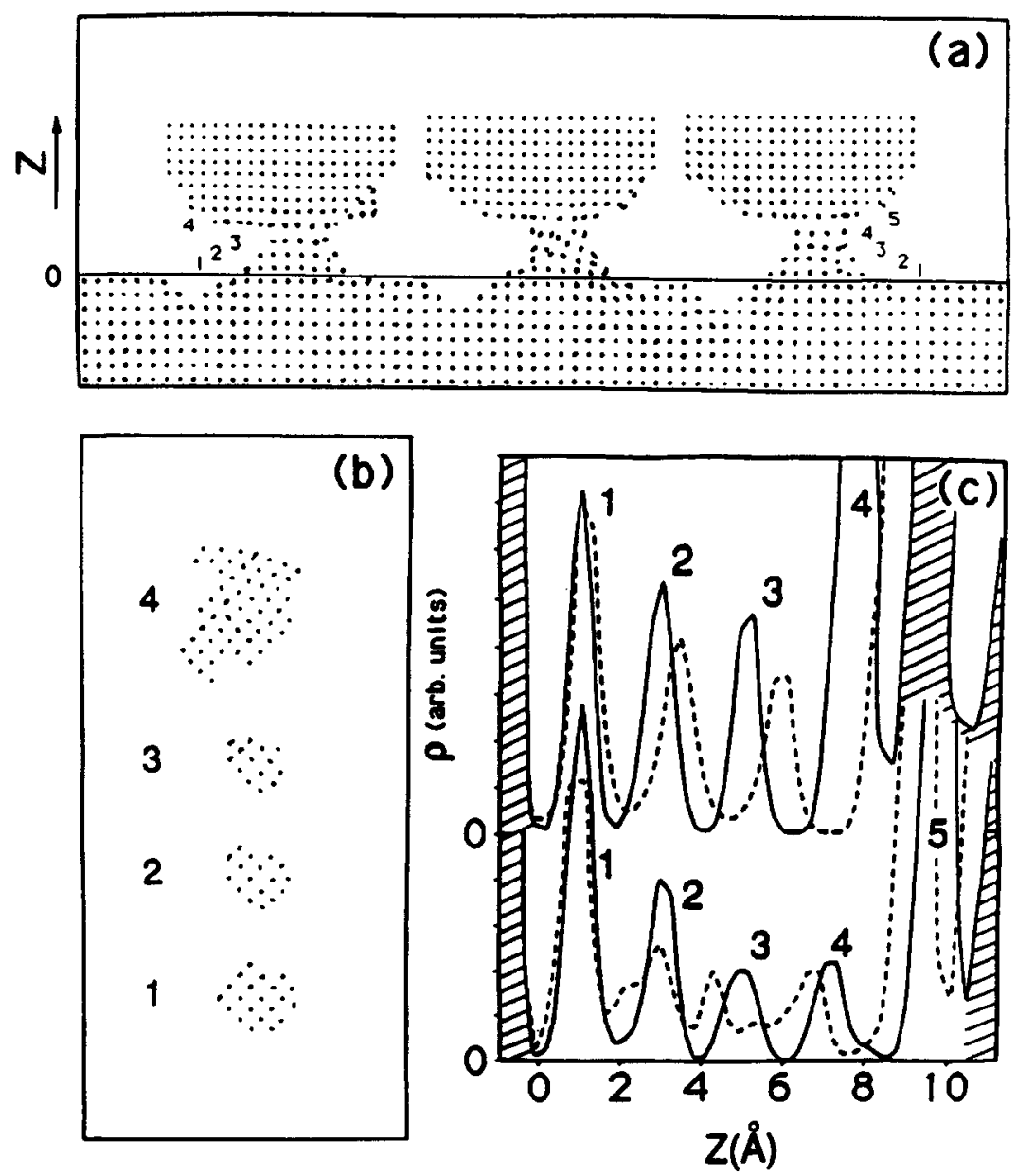

Fig. 10. (a) Side views of atomic configurations obtained from short-time trajectories during a MD simulation [13] of a Ni tip slightly indented into, and then retracted from, an $\mathrm{Au}(001)$ surface at $300 \mathrm{~K}$. On the left, a 4-layer ordered gold junction formed between the tip and the substrate (the 4th layer of the junction coats the bottom of the tip); the middle configuration demonstrates disorder in the junction during elongation, culminating in the 5-layer ordered junction shown on the right. (b) Top views of the in-layer atomic arrangements corresponding to the 4-layer ordered wire. (c) Profiles of atomic densities plotted versus distance $(Z)$ along the axis of the wire. The solid lines at the top and bottom correspond to the 40-layer and 5-layer ordered junctions, respectively (i.e., left and right configurations in (a)). The dashed line at the top part corresponds to the 4-layer strained configuration and the one at the bottom to the disondered structure (middle configuration in (a)) which developed during the elongation process. Hatched regions represent Au substrate $(Z \leqslant 0)$ and Ni tip layers. 


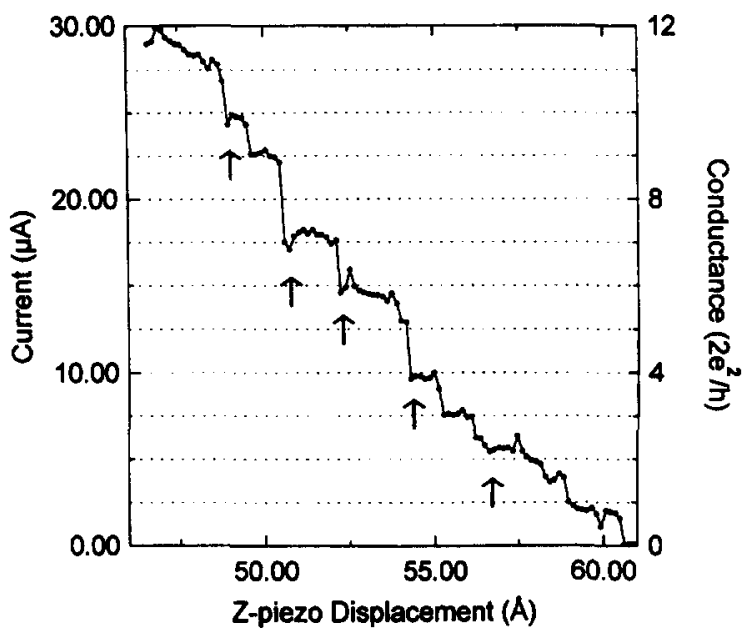

Fig. 11. Current and conductance in a short wire during elongation, exhibiting room-temperature conductance quantization steps. Dashed lines denote $2 e^{2} / h$ intervals. Arrows indicate dips, associated with disordered elongation stages, becoming somewhat less pronounced toward the breaking of the wire [19].

disorder occurring between the intermittent layerordered stages, see Fig. 10). This implies that overall, wires in this length regime maintain a sufficient degree of crystalline order (see Figs. 8 and 10) to sustain quantization of the conductance. Furthermore, significant insights into the microscopic mechanism of the elongation process and nature of disorder in the wire are provided by the observation of dips (local conductance minima) which tend to accompany the quantized conductance steps (see Fig. 11), and can be associated with electron scattering caused by the enhanced structural disorder developing toward the completion of each of the discrete elongation stages of the wire (see Fig. 10). The rapid rise in the conductance following each dip indicates restoration of a higher degree of order in the pulled wire subsequent to the disordering-rearrangement elongation stage. In addition, the variability in the conductance quantization step height (one or two $2 e^{2} / h$ units) and the abovementioned occasional occurrence of two successive steps in an elongation interval of combined length $\sim d$ (see Fig. 11), may originate from stick-slip characteristics of the straining-yielding elongation process and from factors influencing the quantization of transverse electronic stages (channels) in the wire (these include accidental degeneracies of transverse electronic modes, irregular layer shapes (see for example Fig. 10b) which may also lead to energy level spectra characterized by Wigner-like distributions characteristic of quantum chaotic systems, and the likely occurrence of intermediate atomic configurations during the elongation process which satisfy the condition for closing of a conductance channel). These observations support a correlation between the measured patterns and the aforementioned periodic layer-wise order-disorder elongation mechanism of the wire [13].

Simulations of the interactions between the surfaces of crystalline ionic solids $\left(\mathrm{CaF}_{2}\right)$ [16] and between semiconductor surfaces (silicon) $[15,52]$ exhibit similar jump-to-contact and force hysteresis phenomena, with the ionic solids exhibiting a smaller tendency to deform plastically, resulting in brittle failure. In addition, simulations of tribological phenomena when the two interfacing materials slide with respect to one another revealed atomic-scale stick-slip (i.e., oscillatory bonding and bond-breaking behavior) $[15,16,52]$, tip shearing, and interfacial material transfer $[16,50 \mathrm{c}]$, and the results have been used to calculate the critical yield-stress of sheared ionic interfaces and their temperature dependence and correlate these predictions with recent tribo-testing measurements. These simulations [13-16] suggested that the formation of nanowires is a general phenomenon occurring at the latter stages of the elongation process of interfacial contacts, irrespective of the initial size of the contact (in fact for macroscopic contacts multiple junctions are likely to form initially, with only the most ideal ones remaining in the ultimate stages).

Using a similar methodology, atomic-scale mechanisms of friction between diamond (111) surfaces, chosen because of the increasing interest in using diamond as a low-friction wear-resistant coating, have been performed [59]. These studies revealed the atomic-scale nature and mechanisms of excitations and energy transfer, and thus high friction coefficients, for fully hydrogen- or methyl-terminated diamond surfaces, and their dependence on the sliding direction and temperature. Moreover, the reduction of the friction coefficient observed in the presence of chemisorbed longer hydrocarbons, has been correlated at low loads with alignment of the molecules in the sliding direction, and at high loads with diminished corrugation of the underlying surface caused 
by reorientation of the chemisorbed molecular backbones with a tendency to occupy the corrugation troughs in the surface plane.

\subsubsection{Liquid junctions}

It was observed already by the ancients that "Some people put on the ground planed boards because of their smoothness and smear them with grease, because the roughness that is on them is made smooth and so they move the burdens with smaller power" (Heron of Alexandria (c. A.D. 60)). However, the main achievements in lubrication start with the work of Petrov and Tower in 1883 concern- ing friction in railway axle boxes, and the development of the hydrodynamic theory of lubrication by Reynolds in $1886[55,60]$. Current research efforts in this area focus on the way that lubricants inhibit formation of interfacial junctions and on the energetics, structure, dynamics, and rheological and elastohydrodynamic properties of confined complex molecular liquids [51].

Molecular dynamics simulations provide a powerful research tool for studies aimed at exploring the above issues on the atomic scale in an attempt to reveal correlations between the physical properties and response of model lubricants and their molecular
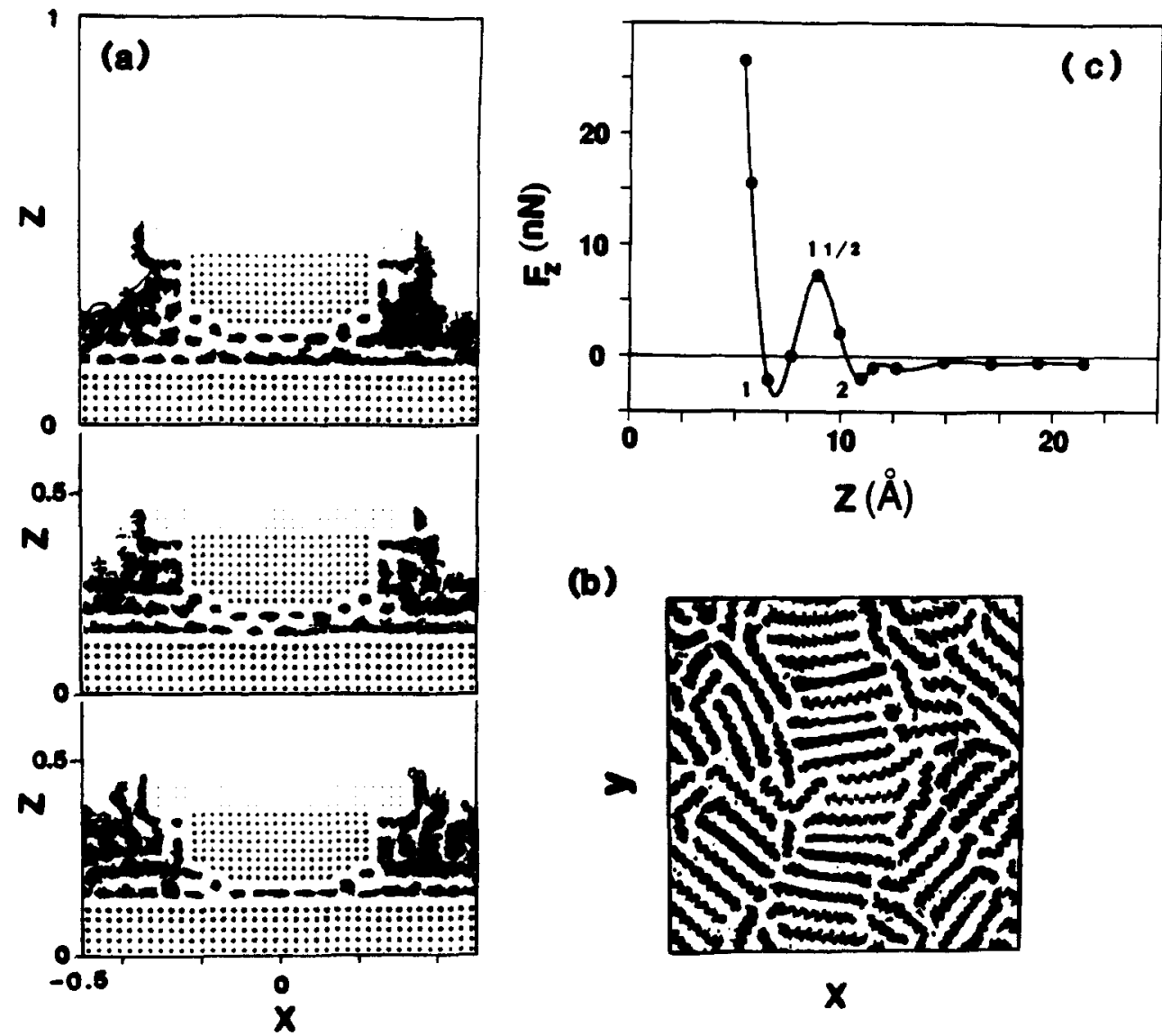

(b)

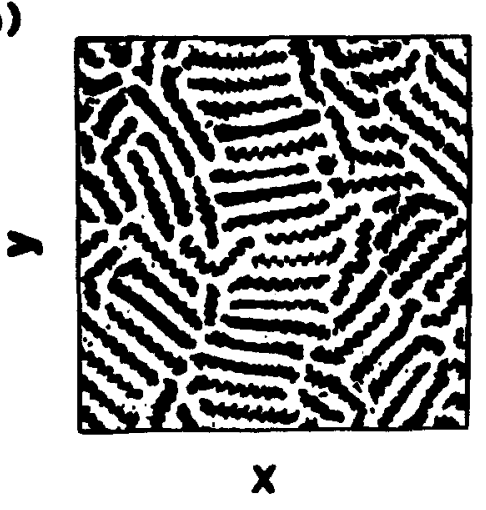

Fig. 12. (a) Side views of atomic configurations of a nickel tip, an $A u(001)$ surface, and a $n-C_{16} H_{34}$ film, starting at a tip-to-surface spacing corresponding to two layers of the confined film $\left(d_{t s}=11 \AA\right.$, top) and ending with a one-layer film $\left(d_{\mathrm{ts}}=6.5 \AA\right.$, bottom). The middle configuration corresponds to a spacing, $d_{t s}=9 \AA$. The linear dimension of the system along the $x$ axis is $77.5 \AA$, and the configurations were obtained in a vertical slice in the $x z$ plane of a width of $16 \AA$. (b) Short-time trajectories of hexadecane molecules in the layer next to the $\mathrm{Au}(001)$ surface, corresponding to the one-layer confined film shown at the bottom of (a). (c) Normal force on the nickel tip (in nN) versus the distance between the tip and the gold surface, $z$ (in $\AA$ ), recorded during the process of lowering of the nickel tip onto the hexadecane-covered $\mathrm{Au}(\mathrm{OO1})$ surface, and illustrating force oscillations associated with the layering transformations in the film [18,70]. 
characteristics, such as chain lengths and molecular structural complexity (i.e., straight versus branched chains). Such $\mathrm{MD}$ simulations have been performed for alkanes and self-assembled boundary lubricant systems, illustrating liquid junction formation [17,18], inhibition of the generation of solid junctions in the presence of thin lubricating films [16] and response characteristics of self-assembled monolayers [61-63] to tip or flat surface compression, layering phenomena and in-plane ordering occurring upon confinement $[17,18,50 c, 64-67]$, collapse and drainage mechanisms of confined molecular films and their dependence on bonding strength to the bounding surfaces [64], the dependence of thin film viscosities on the shear rate [68] and the origins of stick-slip phenomena related to periodic "solidification" and shear melting $[53,54,69]$, mechanisms of cavitation in thin molecular liquid films induced by fast motion of immersed tips [70], elastohydrodynamics in nonuniform junctions [50c], and correlations between structural and dynamical transformations in lubricating films with recorded force-versus-distance characteristics $[17,18,50 \mathrm{c}, 70]$ (Figs. 12 and 13). Additionally, simulations of thin film model lubricants made of mixtures of chain molecules of different lengths, revealed the segmental-sequential molecular mechanisms of preferential surface segregation of the long chain molecules [71]. These simulations provide insights into the structure, rheology and flow characteristics of confined fluids and the dynamics of thin film drainage, and may assist in developing guidelines for the "molecular design" of high performance lubricants.

\section{Epilogue}

To summarize, large-scale computer simulations using advanced interatomic interaction potentials, coupled with novel microscopies, open new avenues for studies of materials, and in particular, the microscopic origins of surface and interfacial phenomena and their macroscopic consequences, such as surface processing via irradiation and phase transformations, the formation of adhesive contacts, tribological phenomena, lubrication and wear, as well as modes and mechanisms of thin film growth of metals and semiconductors [12], surface crystallization of alkane films above the freezing point [72], and the dynamics of collisions of atomic clusters with solid and liquid surfaces $[37,73,74]$. These studies provide significant
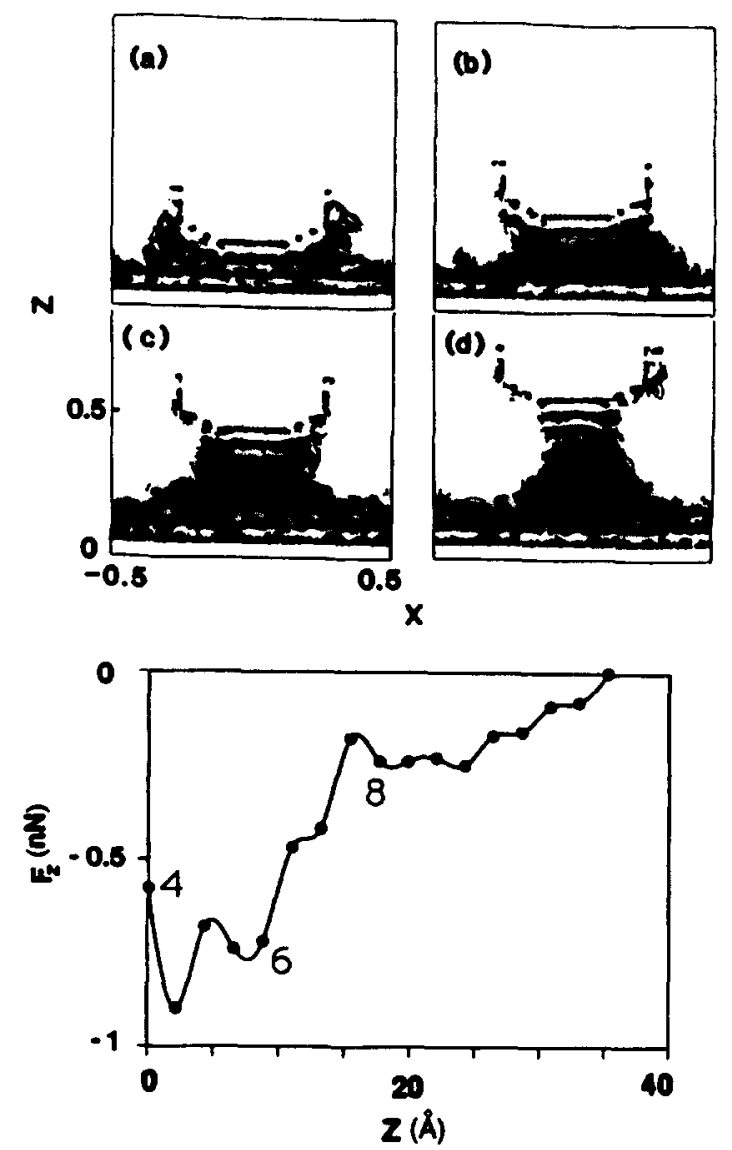

Fig. 13. Short-time trajectories, obtained via MD simulations at $350 \mathrm{~K}[18,70]$, of hexadecane molecules forming a capillary column between a gold substrate and a nickel tip at four stages of the tip-lifting process. Trajectories were plotted in a $23 \AA$ wide slice through the middle of the system. The distances between the tip and the substrate for the configurations shown in (a)-(d) are $d_{\mathrm{ts}}=19.3,28.1,36.9$, and $45.7 \AA$, correspondingly. The length scale of the calculational cell, which was periodically repreated along the $x$ and $y$ directions is $77.5 \AA$. Shown at the bottom is the recorded normal force on the nickel tip (in $\mathrm{nN}$ ) versus the distance between the tip and the gold surface, $z$ (in $\AA$ ) with the origin taken as that corresponding to a 4 layer capillary column (see a). Dots indicate distances for which the system was relaxed during the tip lifting process. The numbers on the graph denote the number of layers in the capillary column (for the eight-layer column the number is ambiguous due to disordering in the middle part, see configuration (c)). The changes in the structural nature of the film upon elongation of the capillary junction are reflected in the variations in $F_{z}$. 
insights into the properties and response characteristics of materials on the atomic and molecular spatial and temporal scales, guide the analysis and design of new experiments, improve theoretical descriptions of the micro- and nano-mechanics and dynamics of materials and provide the foundation for the formulation and implementation of science-based design principles, for the development of new and improved materials and materials processes for technological applications.

Finally, these research endeavors are part of the basic quest to understand and observe natural phenomena with increasing resolution, and of the ever increasing trend towards miniaturization of technological devices, both of which require new techniques and conceptual instruments. As such these studies provide vivid testimony to Feynman's statement: "There is plenty of room at the bottom".

\section{Acknowledgments}

This work is supported by the US Department of Energy, the Air Force Office of Scientific Research, and the National Science Foundation. Simulations were performed on CRAY computers at the National Energy Research Supercomputer Center, Livermore, California, and the Pittsburgh Supercomputer Center.

\section{References}

[1] D. Tabor and R.H. Winterton, Proc. R. Soc. London A 312 (1969) 435.

[2] J.N. Israelachvili and D. Tabor, Nature 241 (1973) 148; J.N. Israelachvili, Acc. Chem. Res. 20 (1987) 415; Proc. Natl. Acad. Sci. USA 84 (1987) 4722; J.N. Israelachvili, P.M. McGuiggan and A.M. Homola, Science 240 (1988) 189.

[3] G. Binning, H. Rohrer, Ch. Gerber and E. Weibel, Phys. Rev. Lett. 50 (1983) 120.

[4] See reviews by: P.K. Hansma and J. Tersoff, J. Appl. Phys. 61 (1986) R1;

J.S. Murday and R.J. Colton, in: Chemistry and Physics of Solid Surfaces, VIII, Eds. R. Vanselow and R. Howe, Springer Series in Surface Science (Springer, Berlin, 1990); Scanning Tunneling Microscopy, Vols. I-III, Eds. R. Wiesendanger and H.J. Guntherodt (Springer, Berlin, 1993).

[5] G. Binning, C.F. Quate and Ch. Gerber, Phys. Rev. Lett. 56 (1986) 930.

[6] D. Sarid, Scanning Force Microscopy (Oxford Univ. Press, New York, 1991).
[7] (a) See articles in: Atomic and Nanometer-Scale Modification of Materials: Fundamentals and Applications, Ed. P. Avouris (Kluwer, Dordrecht, 1993);

(b) See articles in: Computations for the Nano-Scale, Eds. P.E. Blochl, C. Joachim and A.J. Fisher (Kluwer, Dordrecht, 1993).

[8] U. Landman, R.N. Barnett, H.-P. Cheng, C.L. Cleveland and W.D. Luedtke, in: Computations for the Nano-Scale, Eds. P.E. Blochl, C. Joachim and A.J. Fisher (Kluwer, Dordrecht, 1993), and references therein.

[9] See articles in: New Methods for Modeling Processes Within Solids and at Their Surfaces, Eds. C.R.A. Catlow, A.M. Stoneham and J.M. Thomas (Oxford Univ. Press, Oxford, 1993).

[10] H. Hakkinen and U. Landman, Phys. Rev. Lett. 71 (1993) 1023.

[11] W.D. Luedtke and U. Landman, Phys. Rev. Lett. 73 (1994) 569.

[12] U. Landman and W.D. Luedtke, Appl. Surf. Sci. 60/61 (1992) 1.

[13] U. Landman, W.D. Luedtke, N.A. Bumham and R.J. Colton, Science 248 (1990) 454.

[14] U. Landman and W.D. Luedtke, J. Vac. Sci. Technol. 9 (1991) 414

[15] U. Landman, W.D. Luedtke and M.W. Ribarsky, J. Vac. Sci. Technol. A 7 (1989) 2829.

[16] U. Landman, W.D. Luedtke and E.M. Ringer, Wear 153 (1992) 3.

[17] W.D. Luedtke and U. Landman, Comput. Mater. Sci. 1 (1992) 1 .

[18] U. Landman, W.D. Luedtke, J. Ouyang and T.K. Xia, Jpn. J. Appl. Phys. 32 (1993) 1444.

[19] J.I. Pascual, J. Mendez, J. Gomez-Herrero, A.M. Baro, N. Garcia, U. Landman, W.D. Luedtke, E.N. Bogachek and H.-P. Cheng, J. Vac. Sci. Technol. B 13 (1995) 1280; Science 267 (1995) 1783.

[20] J.M. Poate and J. Mayer, Eds., Laser Annealing of Semiconductors (Academic Press, New York, 1982);

C.W. White and P.S. Peercy, Eds., Laser and Electron Beam Processing of Materials (Academic Press, New York, 1980); M. Bass, Laser Materials Processing (North-Holland, Amsterdam, 1993).

[21] For an early Molecular Dynamics Simulation of laser-annealing see, C.L. Cleveland, U. Landman and R.N. Barnett, Phys. Rev. Lett. 49 (1982) 790.

[22] H.E. Elsayed-Ali et al., Phys. Rev. Lett. 58 (1987) 1212.

[23] (a) J.W. Herman and H.E. Elsayed-Ali, Phys. Rev. Lett. 68 (1992) 2952;

(b) J.W. Herman and H.E. Elsayed-Ali, Phys. Rev. Lett. 69 (1992) 1228;

(c) We have chosen copper surfaces for our simulations because of our previous extensive experience with this material.

[24] M. Faraday, Proc. R. Soc. London 10 (1860) 440.

[25] See review by: F. van der Veen, B. Pluis and A.W. Denier van der Gon, in: Kinetics of Ordering at Surfaces, Ed. M.G. Lagally (Plenum, New York, 1990) p. 343. 
[26] R.N. Barnett and U. Landman, Phys. Rev. B 44 (1991) 3226, and references therein.

[27] (a) P. Stoltze, J.K. Norskov and U. Landman, Phys. Rev. Lett. 61 (1988) 440;

P. Stoltze, J. Chem. Phys. 92 (1990) 6306;

(b) H. Hakkinen and M. Manninen, Phys. Rev. B 46 (1992) 1725;

(c) E.T. Chen, R.N. Barnett and U. Landman, Phys. Rev. B 40 (1989) 924;

(d) A. Landa, P. Wynblatt, H. Hakkinen, R.N. Barnett and U. Landman, Phys. Rev. B 51 (1995) 10972.

[28] S.M. Foiles, Phys. Rev. B 32 (1985) 3409.

[29] S.I. Anisimov, B.L. Kapeliovich and T.L. Perelmann, Sov. Phys. JETP 39 (1974) 375, and discussion following Eq. (1) in $\mathrm{it}$;

For the theory of electron-phonon coupling see M.I. Kaganov, I.M. Lifshitz and L.V. Tanatarov, Sov. Phys. JETP 4 (1957) 173.

[30] W.H. Press et al., Numerical Recipes (Cambridge Univ. Press, New York, 1986).

[31] (a) A similar expression was used in the context of studies of energetic displacement cascades by M.W. Finnis, P. Agnew and A.J.E. Foreman, Phys. Rev. B 44 (1991) 567;

(b) For other recent theoretical studies on the same subject see, I. Koponen and M. Hautala, Nucl. Instr. Meth. B 69 (1992) 182;

(c) C.P. Flynn and R.S. Averback, Phys. Rev. B 38 (1988) 7118

A. Caro and M. Victoria, Phys. Rev. A 40 (1989) 2287.

[32] (a) American Institute of Physics Handbook, Ed. D.E. Gray (McGraw-Hill, New York, 1972);

(b) We note that in our simulations of laser irradiation of copper the peak intensities of the 4 ps laser pulses were $I_{0}=5 \times 10^{9}$ and $8 \times 10^{9} \mathrm{~W} / \mathrm{cm}^{2}$, with the former resulting in superheating and the latter in melting of $\mathrm{Cu}(111)$. The superheating pulse corresponds to a total energy of $10^{-2}$ $\mathrm{J} / \mathrm{cm}^{2}$. In the experiments on superheating of $\mathrm{Pb}(111)$ laser pulses of the order of $200 \mathrm{ps}$ with peak intensities of the order of $3.5 \times 10^{7} \mathrm{~W} / \mathrm{cm}^{2}$ were used [23b], corresponding to a total energy of $0.3 \times 10^{-2} \mathrm{~J} / \mathrm{cm}^{2}$. The absorption length used in our simulation $\alpha^{-1}=20 \AA$ and in the experiment on $\mathrm{Pb}(111)$ (with wave length $\lambda=1.16 \mu \mathrm{m}$ ) $\alpha^{-1}=140 \AA$ Taking into account the larger thermal conductivity of $\mathrm{Cu}$ than that of $\mathrm{Pb}(\kappa(\mathrm{Cu}) \approx 5 \kappa(\mathrm{Pb}))$ we estimate that the lattice thermal effects of our pulses are comparable to experimentally realizable conditions.

[33] We note that in the time scale of our interest ( $t<60 \mathrm{ps}$ ), a "quasi-equilibrium" state is reached, where $T_{e}$ and $T_{1}$ remain close to each other, with $T_{1}$ somewhat higher than $T_{e}$, and both slowly equilibrating toward the bulk reference temperature $T_{b}=1100 \mathrm{~K}$.

[34] H. Hakkinen, J. Merikoski and M. Manninen, J. Phys. Condens. Matter 3 (1991) 2755.

[35] For some experimental studies of the stability of nanoscale surface morphological features see: R.C. Jaklevic and L. Elie, Phys. Rev. Lett. 60 (1988) 120;

Y.Z. Li et al., Appl. Phys. Lett. 54 (1989) 1424;
R. Emch et al., J. Appl. Phys. 65 (1989) 79;

D.G. Walmsley et al., Nanostruct. Mater. 3 (1993) 245;

T. Michely et al., Surf. Sci. 230 (1990) L135;

H.J. Mamin et al., Phys. Rev. Lett. 65 (1990) 2418; J. Vac.

Sci. Technol. B 9 (1991) 1398 where stability and minimal diffusion, even at elevated temperatures, are discussed;

D.R. Peale and B.H. Cooper, J. Vac. Sci. Technol. A 10 (1992) 2210;

B.H. Cooper et al., in: Evolution of Surface and Thin Film Microstructure, Eds. H.A. Atwater et al. (MRS, Pittsburgh, 1993) p. 37, where linear in time collapse of gold features on a gold surface in air, and enhanced stability of such features under clean conditions, were observed;

J.J. Pascual et al., Phys. Rev. Lett. 71 (1993) 1852.

[36] With the parameterization given in: J.B. Adams et al., J. Mater. Res. Soc. 4 (1989) 102.

[37] H.-P. Cheng and U. Landman, J. Phys. Chem. 98 (1994) 3527.

[38] For recent experimental studies see: (a) U. Harten et al., Phys. Rev. Lett. 54 (1985) 2619;

(b) Ch. Woll et al., Phys. Rev. B 39 (1989) 7988;

(c) J.V. Barth et al., Phys. Rev. B 42 (1990) 9307;

(d) A.R. Sandy et al., Phys. Rev. B 43 (1991) 4667.

[39] The slip and consequent strain relief involving surface dislocations, involved atoms occupying bridge sites of the underlying substrate surface layer (see Figs. 6b, $c$ where slip occurs along fault contours corresponding to bridge sites). However, the degree to which such bridge-site fault directions are easy slip directions depends on their orientation relative to the substrate and on which bridge sites are occupied.

[40] These surface dislocations are the 2D analogues of the known dissociation of the [110] dislocation in fcc crystals (see D. Hull, Introduction to Dislocations (Pergamon, Oxford, 1975));

For a discussion of surface dislocations in the context of reconstruction of $\mathrm{Au}(111)$ and $\mathrm{Pt}(111)$ see: J.V. Barth et al., Phys. Rev. B 42 (1990) 9307;

D.D. Chambliss et al., Phys. Rev. Lett. 66 (1991) 1721;

M. Bott et al., Phys. Rev. Lett. 70 (1993) 1489.

[41] See reviews by: (a) G. Ehrlich, Appl. Phys. A 55 (1992) 403; (b) M.G. Lagally, Phys. Today 46 (1993) 24.

[42] D.W. Bassett, J. Phys. C 9 (1976) 2491.

[43] S.C. Wang and G. Ehrlich, Surf. Sci. 239 (1990) 301.

[44] G.L. Kellogg, Appl. Surf. Sci. 67 (1993) 134.

[45] For early analytical and simulation studies of dimer diffusion see: U. Landman, M.F. Schlesinger and E.W. Montroll, Phys. Rev. Lett. 38 (1977) 285;

U. Landman and M.F. Schlesinger, Phys. Rev. B 16 (1977) 3389 ;

U. Landman, C.L. Cleveland and R.H. Rast, J. Vac. Sci. Technol. 3 (1985) 1574;

U. Landman and R.H. Rast, in: Dynamics on Surfaces, Eds. B. Pullman, J. Jortner, A. Nitzan and B. Gerber (Reidel, Boston, 1984).

[46] G. Dominique, Surf. Sci. 137 (1984) L103.

[47] A.F. Voter, Phys. Rev. B 34 (1986) 6819. 
[48] S. Stoyanov and H. Muller-Krumbhaar, Surf. Sci. 159 (1985) 49.

[49] C.-L. Liu and J.B. Adams, Surf. Sci. 268 (1992) 73.

[50] (a) See reviews in: Fundamentals of Friction: Macroscopic and Microscopic Processes, Eds. J.L. Singer and H.M. Pollock (Kluwer, Dordrecht, 1991).

(b) See, U. Landman, W.D. Luedtke and E.M. Ringer, in: Fundamentals of Friction: Macroscopic and Microscopic Processes, Eds. J.L. Singer and H.M. Pollock (Kluwer, Dordrecht, 1991).

(c) J. Gao, W.D. Luedtke and U. Landman, Science 270 (1995) 605; Langmuir, in press (1996).

[51] B. Bhushan, J.N. Israelachvili and U. Landman, Nature 374 (1995) 607.

[52] U. Landman, W.D. Luedtke and A. Nitzan, Surf. Sci. 210 (1989) $\mathrm{L} 177$.

[53] P.A. Thompson and M.O. Robbins, Science 250 (1990) 792.

[54] P.A. Thompson, M.O. Robbins and Grest in: Computations for the Nano-Scale, Eds. C. Joachim and A.J. Fisher (Kluwer, Dordrecht, 1993) p. 127, and references therein.

[55] D. Dowson, History of Tribology (Longman, London, 1979).

[56] F.P. Bowden and D. Tabor, Friction (Anchor Press/Doubleday, Garden City, NY, 1973).

[57] A. Stalder and U. Durig, (1994), preprint; see also N. Agrait, G. Rubio and S. Vieira, Phys. Rev. Lett. 74 (1995) 3995, where measurements of $\mathrm{Au}$ wires at $4.2 \mathrm{~K}$, yielding results in agreement with the earlier predictions from simulations, are described.

[58] (a) J.I. Pascual, J. Mendez, J. Gomez-Herrero, A.M. Baro, N. Garcia and V.T. Binh, Phys. Rev. Lett. 71 (1993) 1852. For measurements on other metals, see L. Olesen et al., Phys. Rev. Lett. 72 (1994) 2251.

[59] J.A. Harrison, C.T. White, R.J. Colton and D.W. Brener, MRS Bull. 17 (1993) 50.

[60] See, D. Dowson, in: Fundamentals of Friction: Macroscopic and Microscopic Processes, Eds. J.L. Singer and H.M. Pollock (Kluwer, Dordrecht, 1991).

See also, D. Dowson, History of Tribology (Longman, London, 1979).

[61] K.J. Tupper, R.J. Colton and D.W. Brenner, Langmuir 10 (1994) 2041;

K.J. Tupper and D.W. Brenner, Langmuir 10 (1994) 2335.

[62] M. Callaway, D.J. Tildesley and N. Quirke, Langmuir (1994).

[63] S. Karaborni, Phys. Rev. Lett. 73 (1994) 1668.

[64] M.W. Ribarsky and U. Landman, J. Chem. Phys. 97 (1992) 1937.

[65] M. Schoen, C.L. Rhykerd, D.J. Diestler and J.H. Cushman, Science 245 (1989) 1223.

[66] Y. Wang, K. Hill and J.G. Harris, J. Chem. Phys. 100 (1994) 3276.

[67] S. Gupta, D.C. Koopman, G.B. Westermann-Clark and I.A. Bitsanis, J. Chem. Phys. 100 (1994) 8444.

[68] P.A. Thompson, G.S. Grest and M.O. Robbins, Phys. Rev. Lett. 68 (1992) 3448.

[69] B.N.J. Persson, Phys. Rev. B 50 (1994) 4771.

[70] U. Landman and W.D. Luedtke, MRS Bull. 17 (1993) 36.

[71] T.K. Xia and U. Landman, Science 261 (1993) 1310.

[72] T.K. Xia and U. Landman, Phys. Rev. B 48 (1993) 11313.

[73] C.L. Cleveland and U. Landman, Science 257 (1992) 355.

[74] H.-P. Cheng and U. Landman, Science 260 (1993) 1304. 